\title{
Review on an ethnomedicinal practices of wild mushrooms by the local tribes of India
}

\author{
Sanjit Debnath ${ }^{1 *}$, Bapi Debnath ${ }^{1}$, Panna Das ${ }^{2}$, Ajay Krishna Saha ${ }^{1}$ \\ ${ }^{1}$ Department of Botany, Mycology and Plant Pathology Laboratory, Tripura University, Suryamaninagar, India. \\ ${ }^{2}$ Department of Botany, Microbiology Laboratory, Tripura University, Suryamaninagar, India.
}

\begin{tabular}{l}
\hline ARTICLE INFO \\
\hline Received on: $23 / 02 / 2019$ \\
Accepted on: 03/06/2019 \\
Available online: 03/08/2019
\end{tabular}

\section{Key words:}

Ethnomycology,

ethnomedicine, traditional

mushrooms, ethnic tribes.

\begin{abstract}
Ethnic tribes use wild mushrooms as their traditional medicine as well as food items from ancient times throughout the world and its diverse uses will be helpful to prove different medicinal characterization. The main motive for documentation of this review work is to focus the ethnomedicinally important wild mushrooms of India, which are used by the tribal or local people of India along with their traditional names, various uses and preparation techniques for medical treatments. This review work out to display that there are almost 18 research reports on traditional use of wild mushrooms as medicine from 14 states of India. This documentation implied that at present there are 100 species of macrofungi which are belonged to 56 genera are used by the tribes or local people of India and they use them for common illness, various scared diseases, private diseases and also use as herbal medicines. Present findings exhibit that there are almost 24 modes of preparation for different ethnomedicinal uses. Therefore, there is an urgent necessity to document indigenous knowledge about wild medicinal mushrooms which are used by the tribal peoples belonging to different states of India as well as all over the world.
\end{abstract}

\section{INTRODUCTION}

Mushrooms have been well known to us as an ingredient of gourmet cuisine; chiefly, for their particular flavor as well as various traditional practices and have been valued by humankind as a culinary wonder. Regarding the diversity of fungi, Hawksworth (2001) estimated the occurrence of approximately 1.5 million species worldwide. Ethnomycology is the study of wild macrofungi in folklore and rituals, from prehistoric times to that time (Charaya and Mehrotra, 1999) with their morphometric identification by the tribe along with documentation of nutritional properties and indigenous knowledge regarding their uses as food, medicine and in some other cultural traditional uses also. Edible and medicinal practices of mushrooms in India is quite common, some of which dates back to $1700-1100 \mathrm{BC}$ (Wasson, 1971). India is a diverse country which

\section{*Corresponding Author}

Sanjit Debnath, Department of Botany, Mycology and Plant Pathology

Laboratory, Tripura University, Suryamaninagar, India. E-mail:

sanjitdebnath2888@gmail.com belongs to different types of tribal people or ethnic groups and each group has own management practices of natural resources for their daily uses. The eastern countries of Asia documented the traditional information on the utilization of cooked and therapeutic mushrooms had been accepted on generation to generation but it was not so much in India (Panda and Tayung, 2015). Traditional knowledge of wild edible and medicinal mushrooms might have lost all over the world due to lack of documentation. Traditional information of Indian tribal communities has demonstrated to be broad and profound, consuming nearly 283 species of wild macrofungi out of 2,000 species documented world over (Purkayastha and Chandra, 1985). Indigenous communities have been utilizing non-timber forest products by using their ethnomycological knowledge of wild mushrooms collection, preparations with food items and consuming since time immemorial for their different daily uses, i.e., edible, medicinal and have been considered as the secondary food resources (Boa, 2004). Ethnomycological studies revealed that wild edible mushrooms have been reported from Odisha, West Bengal, Assam, Manipur, Nagaland and Arunachal Pradesh of India (Baruah et al., 1971; Sarma et al., 2010; Sing and Sing, 1993; Sing et al., 2002; Tanti et al., 2011). The North Eastern Himalayan Region of India 
is a biodiversity hotspot which covers eight states, viz., Arunachal Pradesh, Manipur, Assam, Meghalaya, Nagaland, Mizoram, Tripura and Sikkim (Myers, 2003) but very lees amount of research have been carried out from different parts of the Northeastern states of India. The negative site of various traditional knowledge is proper identification, addressing poisoning, various utilization process, spreading of traditional knowledge and market value for selling wild mushrooms due to which many people died in various part of the world (Agrahar-Murugkar and Subbulakshmi, 2005; Basumatary and Gogoi, 2016; Khaund and Joshi, 2014; Sarma et al., 2010; Singh and Chhetry, 2010; Paul et al., 2015). In ancient time, the alertness of wild edible macrofungi and their significance to people of the developing countries have often been ignored but it is only in recent years that initiatives on non-wood forest products have begun to clarify their action use and functions in livelihoods (Devi, 2017; Kumar et al., 2014). Systematics of wild macrofungi has accepted more awareness than other endangered aspects like conservation (Kumar et al., 2014).

Mushrooms have been used as an important food items, and it also contain different medicine properties in all over the world because they contains different active constituents, such as polysaccharides, dietary fibers, selenium oligosaccharides, triterpenoids, peptides, proteins, alcohol, phenols, amino acids and mineral elements (Chang and Buswell, 1996; Chang and Miles, 2002; Lakhanpal et al., 2010; Wani et al., 2010; Wasser, 2010). The World Health Organization (WHO) released a report on ethnomedicine, which has maintained its attractiveness all over the world, and its diverse uses, such as traditional therapies and therapeutic techniques, with Chinese, Ayurveda, Arabic, Unani and native medicine which are speedily growing in the industrialized countries (WHO, 2002-2005). Therapeutic applications of macrofungi have been documented in China, Japan, and Korea for centuries, where fungi have been used by tradition for their medicinal properties. Medicinal application of wild mushrooms to maintain human health was recorded as early as $100 \mathrm{AD}$ in China. The ecological data available on some of the taxa are still not enough and they need to be explored from all over the world. The specific goals of this review work are to document the ethnomedicinal wild mushrooms of India, which were used by the local people of India along with their vernacular names, uses and methods of preparation.

\section{RECORDS OF ETHNOMEDICINAL USES OF MUSHROOMS}

The present review work established that people of the various parts of the world collect the mushrooms from the wild condition and consume various types of edible mushrooms as food or prepare with other food items and also use them for treatment of various human diseases. These wild medicinal mushrooms are edible, medicinal, poisonous, edible with medicinal properties and poisonous with medicinal activity and these mushrooms play a considerable role in sustaining the livelihood of the rural people. Chauhan et al., (2014) recorded twelve wild edible macrofungi, viz., Agaricus campestris, Helvella compressa, Morchella conica, Morchella esculenta, Morchella deliciosa, Ramaria botrytis, Lactarius deliciosus, Rhizopogon vulgaris, Sparassis crispa, Gyromitra sp. Hygrophorus sp. and Lycoperdon sp. which were used by the local tribes of Kinnaur district, Himachal Pradesh,
India as food by various modes of preparation. Dutta and Acharya, (2014) found 34 macrofungal species from West Bengal, India which were eat up or used by the locals and tribals peoples among which 31 macrofungi species were found to be edible and 5 were used as traditional medicine, while some of them were used for both the purposes. Srivastava et al. (2011) conducted an ethnobotanical survey for use of Termitomyces species in Gorakhpur forest division of Uttar Pradesh, India and they found that tribal people and forest dwellers use Termitomyces species as food as well as medicinal purposes but other uses were not clearly known. Earlier findings revealed that macrofungi were used as food supplements and also important due to their healing capacities used by the traditional people (Valverde et al., 2015). Hernández-Santiago et al., (2016) documented a sum of 106 macrofungal species which were growing in oak and pine forest, grassland, deciduous tropical forest, among the identified macrofungi 26 species consumed, 18 recorded as toxic, 6 having ludic application, left over 56 species not individually used but 56 species used as food and 28 species as medicine from Southeastern Mexico. Adhikari et al. (2005) also worked on ethnomedicinal knowledge of wild mushrooms from the vicinities of Lumle and Kathmandu valley of Nepal. They found a total of 24 species of which 18 macrofungi were used as culinary, 8 for various medicinal uses and 3 for their other reasons.

Okigbo and Nwatu, (2015) reported six different species of macrofungi which were used by the people of Anambra State, Nigeria for various purposes, such as Daldinia concentrica in cure of stomach upset, Auricularia auricular-judae and Lentinus squarrosulus in medication of infertility and anaemia, Trichobatrachus robustus in remedies of Anaemia and high blood pressure, Termitomyces sp. for curing Anaemia, weakness, and high blood pressure and $V$. volvacea only used for the cure of Anaemia. Ethnomycological study of the use of mushrooms in North central Nigeria reveals that the majority of citizens consume macrofungi mainly due to their nutritional, palatability and medicinal characteristics (Ayodele et al., 2009). Kimn and Song (2014) reported 38 species of wild mushrooms belonging 33 genera, 22 families by the traditional knowledge recorded from the tribal communities and 158 types of practices were classified and ideal families were Tricholomataceae, Pleurotaceae, Polyporaceae and Hymenochaetaceae. This result revealed 24 methods of preparation for the cooked mushrooms, such as soups, teas, simmered and roasted along with different medicinal uses. Each tribe of India use their own methods for preparation of traditional medicine, such as some fungus or fungal spores used directly, some of them are used in powdered form, mixed with water, milk, tea, sugar, butter, chili, egg and oil, mixed with other plant parts and vegetables for various remedies (Table 1).

The various vernacular names, uses and techniques of preparation of wild mushrooms by the various tribes of India as well as other parts of the world were also recorded. They used a vernacular name for each fungus and these names varied among the different tribes. Present data revealed that wild mushroom species from different parts of India are used as food and traditional medicine by the tribal community and this wide diversity of mushroom species may be due to the existence of diverse vegetations and climatic conditions of India. In this review work, we have found that there are almost 18 reports about the traditional use of wild mushrooms as medicine from 
Table 1. Ethnomedicinally used wild mushrooms collected by different tribe or local people of India along with their vernacular name, used for, methods of preparation for use, name of the states of India from where it was collected and references.

\begin{tabular}{|c|c|c|c|c|c|c|c|}
\hline Sl.No. & $\begin{array}{l}\text { Mushroom } \\
\text { names }\end{array}$ & $\begin{array}{l}\text { Vernacular } \\
\text { names }\end{array}$ & $\begin{array}{l}\text { Name of tribe or } \\
\text { local people }\end{array}$ & Used as & Preparations for use & $\begin{array}{l}\text { Reported from } \\
\text { India }\end{array}$ & References \\
\hline 1. & \multirow{2}{*}{$\begin{array}{l}\text { Daldinia } \\
\text { concentrica } \\
\text { (Bolton) Ces. and } \\
\text { De Not }\end{array}$} & Kath chhatu & NM & $\begin{array}{l}\text { Getting relief from burning, itching } \\
\text { and healing minor skin infections. }\end{array}$ & $\begin{array}{l}\text { Powdered fruitbody is mixed } \\
\text { with coconut oil and applied } \\
\text { to skin. }\end{array}$ & West Bengal & $\begin{array}{l}\text { Dutta and } \\
\text { Achariya, } \\
2014\end{array}$ \\
\hline 2. & & $\begin{array}{l}\text { King Alfred's } \\
\text { Cake }\end{array}$ & Paliyar Tribes & $\begin{array}{l}\text { Curing skin irritations and wound } \\
\text { healing. }\end{array}$ & $\begin{array}{l}\text { Used in the form of dried } \\
\text { powder and applied topically for } \\
\text { any wound. }\end{array}$ & Tamilnadu & $\begin{array}{l}\text { Thangaraj et } \\
\text { al., } 2017\end{array}$ \\
\hline 3. & $\begin{array}{l}\text { Schizophyllum } \\
\text { commune Fr. }\end{array}$ & Pakha chhatu & NM & Used as Tonic. & $\begin{array}{l}\text { Fruitbody is pasted and mixed } \\
\text { with water to make soup. }\end{array}$ & West Bengal & $\begin{array}{l}\text { Dutta and } \\
\text { Achariya, } \\
2014\end{array}$ \\
\hline 4. & \multirow{2}{*}{$\begin{array}{l}\text { Termitomyces } \\
\text { clypeatus } \mathrm{R} . \text { Heim }\end{array}$} & $\begin{array}{l}\text { Bali chhatu/ } \\
\text { Kalunge }\end{array}$ & NM & Used for the treatment of pox. & $\begin{array}{l}\text { Fruitbody is pasted and applied } \\
\text { to the affected area. }\end{array}$ & West Bengal & $\begin{array}{l}\text { Dutta and } \\
\text { Achariya, } \\
2014\end{array}$ \\
\hline 5. & & Orto Onth & $\begin{array}{l}\text { Santal, Lodha, and } \\
\text { Dhangar }\end{array}$ & $\begin{array}{l}\text { Used for the treatments of } \\
\text { antihelminthic and chicken pox. }\end{array}$ & NM & $\begin{array}{l}\text { Eastern Lateritic } \\
\text { Part of India }\end{array}$ & $\begin{array}{l}\text { Manna et al. } \\
2014\end{array}$ \\
\hline 6. & \multirow{3}{*}{$\begin{array}{l}\text { Cordyceps } \\
\text { sinensis (Berk.) } \\
\text { Sacc. }\end{array}$} & $\begin{array}{l}\text { Yarsagumba/ } \\
\text { Jeeban booti }\end{array}$ & NM & $\begin{array}{l}\text { Used for the treatment of } \\
\text { aphrodisiac, invigorative, revitaliser } \\
\text { and anti-aging. }\end{array}$ & $\begin{array}{l}\text { Powdered fruitbody is taken in } \\
\text { tea or soups. }\end{array}$ & West Bengal & $\begin{array}{l}\text { Dutta and } \\
\text { Achariya, } \\
2014\end{array}$ \\
\hline 7. & & Yarsa gumba & Bhutia community & $\begin{array}{l}\text { Remedies of various diseases like, } \\
\text { diabetes and other wasting diseases, } \\
\text { cancer, sexual potency and desire, } \\
\text { etc. }\end{array}$ & $\begin{array}{l}\text { People of both sexes usually } \\
\text { take one piece of } C \text {. Sinensis } \\
\text { with a cup of milk to enhance } \\
\text { their sexual potency and desire. } \\
\text { The Bhutia community put one } \\
\text { piece of } C \text {. sinensisin a cup of } \\
\text { local-made alcohol (chang), } \\
\text { leave it for } 1 \text { hour, and drink it } \\
\text { morning and evening as a tonic. } \\
\text { Some use hot water instead of } \\
\text { alcohol. Some folk healers use } \\
\text { C. Sinensis for diabetes and } \\
\text { other wasting diseases. It is used } \\
\text { for cancer mixed with texus leaf } \\
\text { and Ginseng root decoction. }\end{array}$ & North Sikkim & $\begin{array}{l}\text { Panda and } \\
\text { Swain, } 2011\end{array}$ \\
\hline 8. & & $\begin{array}{l}\text { Sonajhuri } \\
\text { chhatu/ Bomb } \\
\text { chhatu }\end{array}$ & NM & $\begin{array}{l}\text { Getting relief from burning, itching } \\
\text { and healing minor skin infections. }\end{array}$ & $\begin{array}{l}\text { Powdered fruitbody is mixed } \\
\text { with coconut oil and applied } \\
\text { to skin. }\end{array}$ & West Bengal & $\begin{array}{l}\text { Dutta and } \\
\text { Achariya, } \\
2014\end{array}$ \\
\hline 9. & \multirow[t]{2}{*}{$\begin{array}{l}\text { Pisolithus arhizus } \\
\text { (Scop.) Rauschert }\end{array}$} & Tumbe Onth & $\begin{array}{l}\text { Santal, Lodha, and } \\
\text { Dhangar }\end{array}$ & $\begin{array}{l}\text { Yellow-colored spores are used } \\
\text { as medicine for the treatment of } \\
\text { wounds. }\end{array}$ & NM & $\begin{array}{l}\text { Eastern Lateritic } \\
\text { Part of India }\end{array}$ & $\begin{array}{l}\text { Manna et } \\
\text { al.,2014 }\end{array}$ \\
\hline 10. & & $\begin{array}{l}\text { Sonajhuri } \\
\text { Chhatu }\end{array}$ & NM & $\begin{array}{l}\text { Used to cure skin to relieve } \\
\text { burning, itching and healing minor } \\
\text { infections }\end{array}$ & $\begin{array}{l}\text { After being dried and powdered } \\
\text { is mixed with coconut oil. }\end{array}$ & West Bengal & $\begin{array}{l}\text { Dutta and } \\
\text { Achariya, } \\
2014\end{array}$ \\
\hline 11. & \multirow[t]{4}{*}{$\begin{array}{l}\text { Calvatia } \\
\text { gigantean (Batsch } \\
\text { ex Pers.) Lloyd }\end{array}$} & $\mathrm{NM}$ & Paliyar tribes & $\begin{array}{l}\text { Curing stomach upset and to cure } \\
\text { stomach pains in woman during } \\
\text { menstruation. }\end{array}$ & NM & Tamilnadu & $\begin{array}{l}\text { Thangaraj et } \\
\text { al., } 2017\end{array}$ \\
\hline 12. & & NM & Paliyar tribes & $\begin{array}{l}\text { To treat male infertility and male } \\
\text { visceral organ infections. }\end{array}$ & NM & Tamilnadu & $\begin{array}{l}\text { Thangaraj et } \\
\text { al.,2017 }\end{array}$ \\
\hline 13. & & $\begin{array}{l}\text { Bhoroan Pihiri } \\
\text { /Doda }\end{array}$ & Baiga and Bharia. & $\begin{array}{l}\text { The Baiga tribes in cases of partial } \\
\text { paralysis and also used as tonic for } \\
\text { weakness. }\end{array}$ & $\begin{array}{l}\text { A preparation is made by } \\
\text { grinding up sun-dried fruit- } \\
\text { bodies, leaves of Oscium sp., } \\
\text { black pepper and salt. This is } \\
\text { taken either as a powder or in } \\
\text { pea-sized tablets, three times a } \\
\text { day with a glass of cow's milk, } \\
\text { for three months. The Bharia } \\
\text { boil this fungus and take a } \\
\text { tablespoonful of the extract } \\
\text { twice a day before meals. }\end{array}$ & $\begin{array}{l}\text { Jabalpur, } \\
\text { Madhya Pradesh. }\end{array}$ & $\begin{array}{l}\text { Rai et al., } \\
1993\end{array}$ \\
\hline 14. & & $\begin{array}{l}\text { Sita-adim } \\
\text { (small adim }\end{array}$ & Dangi people & $\begin{array}{l}\text { It is consumed to attain good health } \\
\text { specifically during the monsoon or } \\
\text { during convalescence. }\end{array}$ & NM & Gujarat & $\begin{array}{l}\text { Lahiri et al. } \\
2010\end{array}$ \\
\hline
\end{tabular}




\begin{tabular}{|c|c|c|c|c|c|c|c|}
\hline Sl.No. & $\begin{array}{l}\text { Mushroom } \\
\text { names }\end{array}$ & $\begin{array}{l}\text { Vernacular } \\
\text { names }\end{array}$ & $\begin{array}{l}\text { Name of tribe or } \\
\text { local people }\end{array}$ & Used as & Preparations for use & $\begin{array}{l}\text { Reported from } \\
\text { India }\end{array}$ & References \\
\hline 15. & $\begin{array}{l}\text { Podaxis pistillaris } \\
\text { (L.) Fr. }\end{array}$ & NM & Paliyar tribes & $\begin{array}{l}\text { To cure skin diseases and skin } \\
\text { burns. }\end{array}$ & NM & Tamilnadu & $\begin{array}{l}\text { Thangaraj et } \\
\text { al., } 2017\end{array}$ \\
\hline 16. & & $\begin{array}{l}\text { Put- puta/ } \\
\text { Roogda } \\
\text { (some-thing } \\
\text { that burst) }\end{array}$ & $\begin{array}{l}\text { Scheduled Caste, } \\
\text { Brahmin, Kshatriya } \\
\text { and Nepali }\end{array}$ & Spore mass used as burn remedy. & $\begin{array}{l}\text { Spore mass is used directly at } \\
\text { the affected area. }\end{array}$ & $\begin{array}{l}\text { Chakrata, } \\
\text { Dehradun. }\end{array}$ & $\begin{array}{l}\text { Kumar et al., } \\
2017\end{array}$ \\
\hline 17. & $\begin{array}{l}\text { Astraeus } \\
\text { hygrometricus } \\
\text { [Pers] Morgan }\end{array}$ & Savan Putpura & Baiga and Bharia. & An ointment for burn cases. & $\begin{array}{l}\text { The spore mass mixed 1:1 with } \\
\text { mustard seed oil (Brassica } \\
\text { campestris). }\end{array}$ & $\begin{array}{l}\text { Jabalpur, } \\
\text { Madhya Pradesh. }\end{array}$ & $\begin{array}{l}\text { Rai et al., } \\
1993\end{array}$ \\
\hline 18. & & Mati tara & $\begin{array}{l}\text { Kharia, Mankidi, } \\
\text { Santal, Kolha, Munda, } \\
\text { Bhumija, Bhuyan, } \\
\text { Bathudi, Kudumi, Ho, } \\
\text { Mankidia and Birhor, }\end{array}$ & $\begin{array}{l}\text { The spore mass is blended with } \\
\text { mustard seed oil, and used as } \\
\text { a salve against burns. Act as a } \\
\text { haemostatic agent }\end{array}$ & NM & $\begin{array}{l}\text { Northern } \\
\text { Odisha. }\end{array}$ & $\begin{array}{l}\text { Panda and } \\
\text { Tayung, } 2015\end{array}$ \\
\hline 19. & Auricularia & $\begin{array}{l}\text { Kanode/ } \\
\text { Kanchatta (Ear } \\
\text { mushroom) }\end{array}$ & $\begin{array}{l}\text { Scheduled Caste, } \\
\text { Brahmin, Kshatriya } \\
\text { and Nepali }\end{array}$ & The ear puss. & $\begin{array}{l}\text { The mushroom is dried and } \\
\text { ground and mixed in some } \\
\text { liquid. }\end{array}$ & $\begin{array}{l}\text { Chakrata, } \\
\text { Dehradun. }\end{array}$ & $\begin{array}{l}\text { Kumar et al., } \\
2017\end{array}$ \\
\hline 20. & $\begin{array}{l}\text { auricula-judae } \\
\text { (Bull.) Quél. }\end{array}$ & Rudh Papad. & $\begin{array}{l}\text { Local people of } \\
\text { Kashmir }\end{array}$ & $\begin{array}{l}\text { Locally used for colds, sore } \\
\text { throats, sore eyes, hypertension and } \\
\text { jaundice. }\end{array}$ & NM & $\begin{array}{l}\text { Kashmir } \\
\text { Himalayas, India }\end{array}$ & $\begin{array}{l}\text { Pala et al., } \\
2013\end{array}$ \\
\hline 21. & & NM & $\begin{array}{l}\text { Scheduled Caste, } \\
\text { Brahmin, Kshatriya } \\
\text { and Nepali }\end{array}$ & Enhancing the milk secretion. & $\begin{array}{l}\text { The mushroom is dried and } \\
\text { powdered. The powder is } \\
\text { consumed orally with hot water. }\end{array}$ & $\begin{array}{l}\text { Chakrata, } \\
\text { Dehradun }\end{array}$ & $\begin{array}{l}\text { Kumar et al., } \\
2017\end{array}$ \\
\hline 22. & $\begin{array}{l}\text { Ganoderma } \\
\text { lucidum (Curtis) P. }\end{array}$ & NM & Mokokchung & Herbal medicine to cure asthma. & NM & $\begin{array}{l}\text { Nagaland, North } \\
\text { East India }\end{array}$ & $\begin{array}{l}\text { Kumar et al., } \\
2014\end{array}$ \\
\hline 23. & Karst. & Heand & $\begin{array}{l}\text { Local people of } \\
\text { Jammu and Kashmir }\end{array}$ & $\begin{array}{l}\text { Used for recovering from } \\
\text { prolonged illness. }\end{array}$ & As tea against multiple ailments. & $\begin{array}{l}\text { Northern } \\
\text { Districts of } \\
\text { Jammu and } \\
\text { Kashmir. }\end{array}$ & $\begin{array}{l}\text { Malik et al., } \\
2017\end{array}$ \\
\hline 24. & $\begin{array}{l}\text { Hypsizygus } \\
\text { tessellates(Bull.) } \\
\text { Singer }\end{array}$ & NM & Mokokchung & $\begin{array}{l}\text { Herbal medicine to cure skin } \\
\text { diseases. }\end{array}$ & NM & $\begin{array}{l}\text { Nagaland, North } \\
\text { East India }\end{array}$ & $\begin{array}{l}\text { Kumar et al., } \\
2014\end{array}$ \\
\hline 25. & $\begin{array}{l}\text { Entoloma bloxami } \\
\text { (Berk. \& Broome) } \\
\text { Sacc. }\end{array}$ & NM & Mokokchung & $\begin{array}{l}\text { Herbal medicine to cure skin } \\
\text { diseases. }\end{array}$ & NM & $\begin{array}{l}\text { Nagaland, North } \\
\text { East India }\end{array}$ & $\begin{array}{l}\text { Kumar et al., } \\
2014\end{array}$ \\
\hline 26. & $\begin{array}{l}\text { Geastrum triplex } \\
\text { Jungh. }\end{array}$ & NM & $\begin{array}{l}\text { Scheduled Caste, } \\
\text { Brahmin, Kshatriya } \\
\text { and Nepali. }\end{array}$ & Spore mass for burn remedy. & $\begin{array}{l}\text { They appear to be quite effective } \\
\text { when applied with stored rain } \\
\text { water. }\end{array}$ & $\begin{array}{l}\text { Chakrata, } \\
\text { Dehradun }\end{array}$ & $\begin{array}{l}\text { Kumar et al., } \\
2017\end{array}$ \\
\hline 27. & & Phut phuta & $\begin{array}{l}\text { Scheduled Caste, } \\
\text { Brahmin, Kshatriya } \\
\text { and Nepali. }\end{array}$ & Spore mass for burn remedy. & $\begin{array}{l}\text { They appear to be quite effective } \\
\text { when applied with stored rain } \\
\text { water. }\end{array}$ & $\begin{array}{l}\text { Chakrata, } \\
\text { Dehradun }\end{array}$ & $\begin{array}{l}\text { Kumar et al., } \\
2017\end{array}$ \\
\hline 28. & $\begin{array}{l}\text { Lycoperdon } \\
\text { perlatum Pers }\end{array}$ & Nilnass & $\begin{array}{l}\text { Local people of } \\
\text { Kashmir }\end{array}$ & $\begin{array}{l}\text { Used to dress wounds for quick } \\
\text { healing and by bee keepers to } \\
\text { intoxicate the honey bees and } \\
\text { spores are sprinkled on wounds to } \\
\text { stop bleeding. }\end{array}$ & NM & $\begin{array}{l}\text { Kashmir } \\
\text { Himalayas }\end{array}$ & $\begin{array}{l}\text { Pala et al., } \\
2013\end{array}$ \\
\hline 29. & & Shale saes & $\begin{array}{l}\text { Local people of } \\
\text { Jammu and Kashmir }\end{array}$ & $\begin{array}{l}\text { Used for healing of wounds and } \\
\text { frost bites. }\end{array}$ & NM & $\begin{array}{l}\text { Northern } \\
\text { Districts of } \\
\text { Jammu and } \\
\text { Kashmir. }\end{array}$ & $\begin{array}{l}\text { Malik et al., } \\
2017\end{array}$ \\
\hline 30. & & Phut phuta & $\begin{array}{l}\text { Scheduled Caste, } \\
\text { Brahmin, Kshatriya } \\
\text { and Nepali. }\end{array}$ & Spore mass for burn remedy. & $\begin{array}{l}\text { They appear to be quite effective } \\
\text { when applied with stored rain } \\
\text { water. }\end{array}$ & $\begin{array}{l}\text { Chakrata, } \\
\text { Dehradun }\end{array}$ & $\begin{array}{l}\text { Kumar et. al, } \\
2017\end{array}$ \\
\hline 31. & $\begin{array}{l}\text { Lycoperdon } \\
\text { pyriforme Schaeff. }\end{array}$ & Shale saes & $\begin{array}{l}\text { Local people of } \\
\text { Jammu and Kashmir }\end{array}$ & $\begin{array}{l}\text { Used for healing of wounds and } \\
\text { frost bites. }\end{array}$ & NM & $\begin{array}{l}\text { Northern } \\
\text { Districts of } \\
\text { Jammu and } \\
\text { Kashmir. }\end{array}$ & $\begin{array}{l}\text { Malik et al., } \\
2017\end{array}$ \\
\hline 32. & & Nilnass & $\begin{array}{l}\text { Local people of } \\
\text { Kashmir }\end{array}$ & $\begin{array}{l}\text { Used it for treatment of frostbite } \\
\text { and sprinkle spores on wounds to } \\
\text { stop bleeding. }\end{array}$ & NM & $\begin{array}{l}\text { Kashmir } \\
\text { Himalayas }\end{array}$ & $\begin{array}{l}\text { Pala et al., } \\
2013\end{array}$ \\
\hline
\end{tabular}




\begin{tabular}{|c|c|c|c|c|c|c|c|}
\hline Sl.No. & $\begin{array}{l}\text { Mushroom } \\
\text { names }\end{array}$ & $\begin{array}{l}\text { Vernacular } \\
\text { names }\end{array}$ & $\begin{array}{l}\text { Name of tribe or } \\
\text { local people }\end{array}$ & Used as & Preparations for use & $\begin{array}{l}\text { Reported from } \\
\text { India }\end{array}$ & References \\
\hline 33. & Phallus sp. & Stinkhorn & $\begin{array}{l}\text { Scheduled Caste, } \\
\text { Brahmin, Kshatriya } \\
\text { and Nepali. }\end{array}$ & Aphrodisiac. & NM & $\begin{array}{l}\text { Chakrata, } \\
\text { Dehradun }\end{array}$ & $\begin{array}{l}\text { Kumar et al., } \\
2017\end{array}$ \\
\hline 34. & Stereum sp. & NM & $\begin{array}{l}\text { Scheduled Caste, } \\
\text { Brahmin, Kshatriya } \\
\text { and Nepali. }\end{array}$ & Healing wounds. & $\begin{array}{l}\text { Dried fruitbodies of the } \\
\text { mushroom is turned into } \\
\text { powdered form and paste is } \\
\text { prepared. }\end{array}$ & $\begin{array}{l}\text { Chakrata, } \\
\text { Dehradun }\end{array}$ & $\begin{array}{l}\text { Kumar et al., } \\
2017\end{array}$ \\
\hline 35. & Truffle (Tuber sp.) & Jhanda & $\begin{array}{l}\text { Scheduled Caste, } \\
\text { Brahmin, Kshatriya } \\
\text { and Nepali }\end{array}$ & Mouth freshener. & NM & $\begin{array}{l}\text { Chakrata, } \\
\text { Dehradun }\end{array}$ & $\begin{array}{l}\text { Kumar et al., } \\
2017\end{array}$ \\
\hline 36. & Agaricus sp. & Gobari Pihiri & Baiga & Remedy for goitres. & $\begin{array}{l}\text { Baiga people hang sundried } \\
\text { fruit-bodies of this mushroom } \\
\text { round the neck. }\end{array}$ & $\begin{array}{l}\text { Jabalpur, } \\
\text { Madhya Pradesh. }\end{array}$ & $\begin{array}{l}\text { Rai et al., } \\
1993\end{array}$ \\
\hline 37. & $\begin{array}{l}\text { Bovista pusilla } \\
\text { (Batsch) Pers. }\end{array}$ & Phusphush & Baiga & Staunch bleeding of cuts. & NM & $\begin{array}{l}\text { Jabalpur, } \\
\text { Madhya Pradesh. }\end{array}$ & $\begin{array}{l}\text { Rai et al., } \\
1993\end{array}$ \\
\hline 38. & $\begin{array}{l}\text { Calvatia } \\
\text { cyathiformis } \\
\text { (Bosc) Morgan }\end{array}$ & Dharti Phool & Baiga and Bharia. & $\begin{array}{l}\text { Healing wounds and to check pus } \\
\text { formation. }\end{array}$ & NM & $\begin{array}{l}\text { Jabalpur, } \\
\text { Madhya Pradesh }\end{array}$ & $\begin{array}{l}\text { Rai et al., } \\
1993\end{array}$ \\
\hline 39. & $\begin{array}{l}\text { Cyathus } \\
\text { stercoreus (Schw.) } \\
\text { de Toni }\end{array}$ & Nirghunti & Baiga. & $\begin{array}{l}\text { Sore (pain, redness, conjunctivitis) } \\
\text { eyes. }\end{array}$ & $\begin{array}{l}\text { The peridioles are ground up } \\
\text { with water, filtered through } \\
\text { cotton, and used as eye-drops, } \\
\text { two drops twice a day. }\end{array}$ & $\begin{array}{l}\text { Jabalpur, } \\
\text { Madhya Pradesh }\end{array}$ & $\begin{array}{l}\text { Rai et al., } \\
1993\end{array}$ \\
\hline 40. & $\begin{array}{l}\text { Cyathus limbatus } \\
\text { Tul. }\end{array}$ & Kulhari & Bharia. & $\begin{array}{l}\text { Soothing eye disorders (pain, } \\
\text { redness, conjunctivitis). }\end{array}$ & $\begin{array}{l}\text { The peridioles are ground up } \\
\text { with water, filtered through } \\
\text { cotton, and used as eye-drops, } \\
\text { two drops twice a day }\end{array}$ & $\begin{array}{l}\text { Jabalpur, } \\
\text { Madhya Pradesh }\end{array}$ & $\begin{array}{l}\text { Rai et al., } \\
1993\end{array}$ \\
\hline \multirow[t]{2}{*}{41.} & $\begin{array}{l}\text { Microporus } \\
\text { xanthopus (Fr.) } \\
\text { Kuntze }\end{array}$ & & Baiga and Bharia. & $\begin{array}{l}\text { A remedy against fever and } \\
\text { vomiting, and for the relief of } \\
\text { earache. }\end{array}$ & $\begin{array}{l}\text { Ground up with the gum of } \\
\text { Pterocarpum marsupium, one } \\
\text { teaspoonful with a glass of } \\
\text { water, three times a day. }\end{array}$ & & $\begin{array}{l}\text { Rai et al., } \\
1993\end{array}$ \\
\hline & & Saja Pihiri & & & $\begin{array}{l}\text { The Bharia people grind it with } \\
\text { water and filter it. }\end{array}$ & $\begin{array}{l}\text { Jabalpur, } \\
\text { Madhya Pradesh. }\end{array}$ & \\
\hline 42. & $\begin{array}{l}\text { Phallus } \\
\text { rubicundus } \\
\text { (Bosc.) Fr }\end{array}$ & Jhiri Pihiri & Baiga and Bharia & $\begin{array}{l}\text { Against typhoid and for the relief of } \\
\text { labour pain. }\end{array}$ & $\begin{array}{l}\text { The fungus is ground up and } \\
\text { mixed with old sugar-cake. One } \\
\text { full teaspoonful three times } \\
\text { a day. }\end{array}$ & $\begin{array}{l}\text { Jabalpur, } \\
\text { Madhya Pradesh. }\end{array}$ & $\begin{array}{l}\text { Rai et al., } \\
1993\end{array}$ \\
\hline 43. & $\begin{array}{l}\text { Xylaria } \\
\text { polymorpha } \\
\text { [Pers.] Grev. }\end{array}$ & Phoot Dooth & Baiga and Bharia. & To induce lactation. & $\begin{array}{l}\text { The fungus is ground to a fine } \\
\text { powder, mixed 1:1 with old } \\
\text { sugar-cake, and formed into pea- } \\
\text { sized tablets. These are taken } \\
\text { twice a day before meals for five } \\
\text { days with a glass of cow's milk. }\end{array}$ & $\begin{array}{l}\text { Jabalpur, } \\
\text { Madhya Pradesh. }\end{array}$ & $\begin{array}{l}\text { Rai et al., } \\
1993\end{array}$ \\
\hline 44. & Volvariella sp. & Kuta Chatu & $\begin{array}{l}\text { Kharia, Mankidi, } \\
\text { Santal, Kolha, Munda, } \\
\text { Bhumija, Bhuyan, } \\
\text { Bathudi, Kudumi, Ho, } \\
\text { Mankidia and Birhor }\end{array}$ & Lowering high blood pressure. & NM & Northern Odisha. & $\begin{array}{l}\text { Panda and } \\
\text { Tayung, } 2015\end{array}$ \\
\hline 45. & Geastrum sp. & Mati tara & $\begin{array}{l}\text { Kharia, Mankidi, } \\
\text { Santal, Kolha, Munda, } \\
\text { Bhumija, Bhuyan, } \\
\text { Bathudi, Kudumi, Ho, } \\
\text { Mankidia and Birhor, }\end{array}$ & $\begin{array}{l}\text { Reduce staunch bleeding and } \\
\text { reduce swelling }\end{array}$ & NM & Northern Odisha. & $\begin{array}{l}\text { Panda and } \\
\text { Tayung, } 2015\end{array}$ \\
\hline 46. & $\begin{array}{l}\text { Termitomyces } \\
\text { reticulates } \text { Van der } \\
\text { Westh. \& Eicker }\end{array}$ & $\begin{array}{l}\text { Parabana, Ada } \\
\text { chatu }\end{array}$ & $\begin{array}{l}\text { Kharia, Mankidi, } \\
\text { Santal, Kolha, Munda, } \\
\text { Bhumija, Bhuyan, } \\
\text { Bathudi, Kudumi, Ho, } \\
\text { Mankidia and Birhor, }\end{array}$ & $\begin{array}{l}\text { Rheumatism and lowering high } \\
\text { blood pressure. }\end{array}$ & NM & Northern Odisha. & $\begin{array}{l}\text { Panda and } \\
\text { Tayung, } 2015\end{array}$ \\
\hline 47. & Lactarius sp. & Pitha & $\begin{array}{l}\text { Kharia, Mankidi, } \\
\text { Santal, Kolha, Munda, } \\
\text { Bhumija, Bhuyan, } \\
\text { Bathudi, Kudumi, Ho, } \\
\text { Mankidia and Birhor }\end{array}$ & Lower high blood pressure. & NM & Northern Odisha. & $\begin{array}{l}\text { Panda and } \\
\text { Tayung, } 2015\end{array}$ \\
\hline
\end{tabular}




\begin{tabular}{|c|c|c|c|c|c|c|c|}
\hline Sl.No. & $\begin{array}{l}\text { Mushroom } \\
\text { names }\end{array}$ & $\begin{array}{l}\text { Vernacular } \\
\text { names }\end{array}$ & $\begin{array}{l}\text { Name of tribe or } \\
\text { local people }\end{array}$ & Used as & Preparations for use & $\begin{array}{l}\text { Reported from } \\
\text { India }\end{array}$ & References \\
\hline 48. & $\begin{array}{l}\text { Volvariella } \\
\text { glandiformis } \\
\text { (Berk. \& Broome) } \\
\text { Pegler }\end{array}$ & Bali chatu & $\begin{array}{l}\text { Kharia, Mankidi, } \\
\text { Santal, Kolha, Munda, } \\
\text { Bhumija, Bhuyan, } \\
\text { Bathudi, Kudumi, Ho, } \\
\text { Mankidia and Birhor }\end{array}$ & Lower high blood pressure & NM & Northern Odisha. & $\begin{array}{l}\text { Panda and } \\
\text { Tayung, 2015 }\end{array}$ \\
\hline 49. & Lycoperdon sp.1 & Anthua & $\begin{array}{l}\text { Kharia, Mankidi, } \\
\text { Santal, Kolha, Munda, } \\
\text { Bhumija, Bhuyan, } \\
\text { Bathudi, Kudumi, Ho, } \\
\text { Mankidia and Birhor }\end{array}$ & Cure wound. & NM & Northern Odisha. & $\begin{array}{l}\text { Panda and } \\
\text { Tayung, 2015 }\end{array}$ \\
\hline 50. & Lycoperdon sp. 2 & Anthua & $\begin{array}{l}\text { Kharia, Mankidi, } \\
\text { Santal, Kolha, Munda, } \\
\text { Bhumija, Bhuyan, } \\
\text { Bathudi, Kudumi, Ho, } \\
\text { Mankidia and Birhor, }\end{array}$ & Cure wound. & NM & Northern Odisha. & $\begin{array}{l}\text { Panda and } \\
\text { Tayung, } 2015\end{array}$ \\
\hline 51. & Lycoperdon sp. 3 & Jatia rutka & $\begin{array}{l}\text { Kharia, Mankidi, } \\
\text { Santal, Kolha, Munda, } \\
\text { Bhumija, Bhuyan, } \\
\text { Bathudi, Kudumi, Ho, } \\
\text { Mankidia and Birhor }\end{array}$ & Cure wound. & NM & Northern Odisha. & $\begin{array}{l}\text { Panda and } \\
\text { Tayung, 2015 }\end{array}$ \\
\hline 52. & Tuber $\mathrm{sp}$. & Desi rutka & $\begin{array}{l}\text { Kharia, Mankidi, } \\
\text { Santal, Kolha, Munda, } \\
\text { Bhumija, Bhuyan, } \\
\text { Bathudi, Kudumi, Ho, } \\
\text { Mankidia and Birhor }\end{array}$ & Cure wound. & NM & Northern Odisha & $\begin{array}{l}\text { Panda and } \\
\text { Tayung, 2015 }\end{array}$ \\
\hline 53. & Russula sp. & Pija chatu & $\begin{array}{l}\text { Kharia, Mankidi, } \\
\text { Santal, Kolha, Munda, } \\
\text { Bhumija, Bhuyan, } \\
\text { Bathudi, Kudumi, Ho, } \\
\text { Mankidia and Birhor }\end{array}$ & $\begin{array}{l}\text { For the treatments of malnutrition, } \\
\text { weakness and a delicious food item } \\
\text { used by local people. }\end{array}$ & NM & Northern Odisha & $\begin{array}{l}\text { Panda and } \\
\text { Tayung, 2015 }\end{array}$ \\
\hline 54. & $\begin{array}{l}\text { Agaricus augustus } \\
\text { Fries }\end{array}$ & Haend & $\begin{array}{l}\text { Local people of } \\
\text { Jammu and Kashmir }\end{array}$ & $\begin{array}{l}\text { Widely used as food and } \\
\text { general tonic. Prescribed as a } \\
\text { supplementary diet to patients } \\
\text { suffering from asthma, stroke, heart } \\
\text { ailments, and diabetes. }\end{array}$ & NM & $\begin{array}{l}\text { Northern } \\
\text { Districts of } \\
\text { Jammu and } \\
\text { Kashmir. }\end{array}$ & $\begin{array}{l}\text { Malik et al., } \\
2017\end{array}$ \\
\hline 55. & Agaricus bisporus & Haend & $\begin{array}{l}\text { Local people of } \\
\text { Jammu and Kashmir }\end{array}$ & $\begin{array}{l}\text { Widely used as food and general } \\
\text { tonic. }\end{array}$ & NM & $\begin{array}{l}\text { Northern } \\
\text { Districts of } \\
\text { Jammu and } \\
\text { Kashmir. }\end{array}$ & $\begin{array}{l}\text { Malik et al., } \\
2017\end{array}$ \\
\hline 56. & (Lange) Imbach & Maazh hadur & $\begin{array}{l}\text { Local people of } \\
\text { Kashmir }\end{array}$ & $\begin{array}{l}\text { Used for the treatment of } \\
\text { leucoderma and considered } \\
\text { as a tonic for patients with } \\
\text { cardiovascular disease. }\end{array}$ & $\begin{array}{l}\text { Its powder is mixed with butter } \\
\text { and used for the treatment. }\end{array}$ & $\begin{array}{l}\text { Kashmir } \\
\text { Himalayas }\end{array}$ & $\begin{array}{l}\text { Pala et al., } \\
2013\end{array}$ \\
\hline 57. & $\begin{array}{l}\text { Agaricus } \\
\text { campestris }\end{array}$ & Haend & $\begin{array}{l}\text { Local people of } \\
\text { Jammu and Kashmir }\end{array}$ & $\begin{array}{l}\text { General tonic, Immunomodulators. } \\
\text { Prescribed to diabetic patients }\end{array}$ & NM & $\begin{array}{l}\text { Northern } \\
\text { Districts of } \\
\text { Jammu and } \\
\text { Kashmir. }\end{array}$ & $\begin{array}{l}\text { Malik et al., } \\
2017\end{array}$ \\
\hline 58. & L.: Fr. & Maazh hadur & $\begin{array}{l}\text { Local people of } \\
\text { Kashmir. }\end{array}$ & $\begin{array}{l}\text { Used to treat the scalds and burns } \\
\text { by the tribals. }\end{array}$ & $\begin{array}{l}\text { The fresh fruiting body is } \\
\text { crushed and applied to the } \\
\text { affected area. }\end{array}$ & $\begin{array}{l}\text { Kashmir } \\
\text { Himalayas, India }\end{array}$ & $\begin{array}{l}\text { Pala et al., } \\
2013\end{array}$ \\
\hline 59. & $\begin{array}{l}\text { Boletus edulis } \\
\text { Bull. }\end{array}$ & Rate & $\begin{array}{l}\text { Local people of } \\
\text { Jammu and Kashmir }\end{array}$ & $\begin{array}{l}\text { Used after deliveries either alone } \\
\text { or with dandelion as expectorant, } \\
\text { antidepressant, treating lumbago, } \\
\text { leg pains, numbness in limbs and } \\
\text { tendon discomfort. }\end{array}$ & NM & $\begin{array}{l}\text { Northern } \\
\text { Districts of } \\
\text { Jammu and } \\
\text { Kashmir. }\end{array}$ & $\begin{array}{l}\text { Malik et al., } \\
2017\end{array}$ \\
\hline
\end{tabular}




\begin{tabular}{|c|c|c|c|c|c|c|c|}
\hline Sl.No. & $\begin{array}{l}\text { Mushroom } \\
\text { names }\end{array}$ & $\begin{array}{l}\text { Vernacular } \\
\text { names }\end{array}$ & $\begin{array}{l}\text { Name of tribe or } \\
\text { local people }\end{array}$ & Used as & Preparations for use & $\begin{array}{l}\text { Reported from } \\
\text { India }\end{array}$ & References \\
\hline 60. & \multirow{3}{*}{$\begin{array}{l}\text { Bovista plumbea } \\
\text { Pers }\end{array}$} & Badul & $\begin{array}{l}\text { Local people of } \\
\text { Jammu and Kashmir }\end{array}$ & $\begin{array}{l}\text { Used for respiratory tract } \\
\text { infections after boiling in milk and } \\
\text { concentrated to powder form is } \\
\text { used against frost bites. }\end{array}$ & $\begin{array}{l}\text { Cooked in milk with some } \\
\text { preliminary treatment. }\end{array}$ & $\begin{array}{l}\text { Northern } \\
\text { Districts of } \\
\text { Jammu and } \\
\text { Kashmir. }\end{array}$ & $\begin{array}{l}\text { Malik et al. } \\
2017\end{array}$ \\
\hline 61. & & $\begin{array}{l}\text { Mwikhun } \\
\text { daudwi }\end{array}$ & Bodo tribe. & $\begin{array}{l}\text { Used for the treatment of sores, } \\
\text { ulcers and skin infection. }\end{array}$ & From powdered fruiting bodies. & $\begin{array}{l}\text { Chirang District } \\
\text { of Assam, North- } \\
\text { East India. }\end{array}$ & $\begin{array}{l}\text { Devi et al., } \\
2016\end{array}$ \\
\hline 62. & & Sesdeung. & $\begin{array}{l}\text { Local people of } \\
\text { Kashmir }\end{array}$ & $\begin{array}{l}\text { Used for the treatment of frost bite } \\
\text { and to heal wounds. }\end{array}$ & NM & $\begin{array}{l}\text { Kashmir } \\
\text { Himalayas }\end{array}$ & $\begin{array}{l}\text { Pala et al. } \\
2013\end{array}$ \\
\hline 63. & \multirow[t]{2}{*}{$\begin{array}{l}\text { Cantharellus } \\
\text { cibarius } \mathrm{Fr}\end{array}$} & Pueh Heand & $\begin{array}{l}\text { Local people of } \\
\text { Jammu and Kashmir }\end{array}$ & $\begin{array}{l}\text { Used to cure wounds when used in } \\
\text { powdered form, natural tonic, bone } \\
\text { ailments and general weakness. }\end{array}$ & $\begin{array}{l}\text { Powder from the dried fruitbody } \\
\text { is used to heal wounds. }\end{array}$ & $\begin{array}{l}\text { Northern } \\
\text { Districts of } \\
\text { Jammu and } \\
\text { Kashmir. }\end{array}$ & $\begin{array}{l}\text { Malik et al. } \\
2017\end{array}$ \\
\hline 64. & & Harda guech & $\begin{array}{l}\text { Local people of } \\
\text { Jammu and Kashmir }\end{array}$ & $\begin{array}{l}\text { Young mushrooms or the stipe only } \\
\text { is used against respiratory ailments } \\
\text { and against diabetes. }\end{array}$ & $\begin{array}{l}\text { A decoction is prepared from the } \\
\text { young mushrooms. }\end{array}$ & $\begin{array}{l}\text { Northern } \\
\text { Districts of } \\
\text { Jammu and } \\
\text { Kashmir. }\end{array}$ & $\begin{array}{l}\text { Malik et al. } \\
2017\end{array}$ \\
\hline 65. & \multirow[t]{2}{*}{$\begin{array}{l}\text { Coprinus comatus } \\
\text { (O.F.Mull.: Fr.) } \\
\text { Pers. }\end{array}$} & Ajjio & Ghanashia and Rabaris & $\begin{array}{l}\text { It is mostly used for skin related } \\
\text { diseases like lesions, bruised and } \\
\text { infected skin, or for wound healing. }\end{array}$ & $\begin{array}{l}\text { The part of the fruiting body } \\
\text { used in these cases is the cap and } \\
\text { not the stipe, the cap is opened } \\
\text { and the spore bearing gills are } \\
\text { applied to the affected area } \\
\text { directly, which is then bandaged. }\end{array}$ & Gujarat & $\begin{array}{l}\text { Lahiri et al. } \\
2010\end{array}$ \\
\hline 66. & & Setherwat & $\begin{array}{l}\text { Local people of } \\
\text { Kashmir }\end{array}$ & $\begin{array}{l}\text { Its preparations are also } \\
\text { recommended for the treatment of } \\
\text { respiratory disorders. }\end{array}$ & NM & $\begin{array}{l}\text { Kashmir } \\
\text { Himalayas }\end{array}$ & $\begin{array}{l}\text { Pala et al. } \\
2013\end{array}$ \\
\hline 67. & $\begin{array}{l}\text { Disciotis venosa } \\
\text { (Pers.) Arnould }\end{array}$ & Kana Guech & $\begin{array}{l}\text { Local people of } \\
\text { Jammu and Kashmir }\end{array}$ & Used against common cold. & $\begin{array}{l}\text { As culinary preparations with } \\
\text { chilli and eggs. }\end{array}$ & $\begin{array}{l}\text { Northern } \\
\text { Districts of } \\
\text { Jammu and } \\
\text { Kashmir. }\end{array}$ & $\begin{array}{l}\text { Malik et al. } \\
2017\end{array}$ \\
\hline 68. & \multirow[t]{2}{*}{$\begin{array}{l}\text { Flammulina } \\
\text { velutipes (Curt.) } \\
\text { Singer }\end{array}$} & Heand & $\begin{array}{l}\text { Local people of } \\
\text { Jammu and Kashmir }\end{array}$ & $\begin{array}{l}\text { Used as a tonic and } \\
\text { immunomodulator. }\end{array}$ & NM & $\begin{array}{l}\text { Northern } \\
\text { Districts of } \\
\text { Jammu and } \\
\text { Kashmir. }\end{array}$ & $\begin{array}{l}\text { Malik et al. } \\
2017\end{array}$ \\
\hline 69. & & $\begin{array}{l}\text { Hendh or } \\
\text { Drubdi }\end{array}$ & $\begin{array}{l}\text { Local people of } \\
\text { Kashmir }\end{array}$ & $\begin{array}{l}\text { It is used for diabetes patients by } \\
\text { local herbalists }\end{array}$ & NM & $\begin{array}{l}\text { Kashmir } \\
\text { Himalayas }\end{array}$ & $\begin{array}{l}\text { Pala et al., } \\
2013\end{array}$ \\
\hline 70. & \multirow{2}{*}{$\begin{array}{l}\text { Fomes } \\
\text { fomentarius } \\
\text { (L.) Fr. }\end{array}$} & Heand & $\begin{array}{l}\text { Local people of } \\
\text { Jammu and Kashmir }\end{array}$ & $\begin{array}{l}\text { Used against skin ailments and } \\
\text { also for hypertensive patients. }\end{array}$ & $\begin{array}{l}\text { In case of skin disease its ash } \\
\text { is mixed with oil. Small slices } \\
\text { are prescribed in vegetables to } \\
\text { hypertensive patients. }\end{array}$ & $\begin{array}{l}\text { Northern } \\
\text { Districts of } \\
\text { Jammu and } \\
\text { Kashmir. }\end{array}$ & $\begin{array}{l}\text { Malik et al. } \\
2017\end{array}$ \\
\hline 71. & & Frassi lashe & $\begin{array}{l}\text { Local people of } \\
\text { Kashmir }\end{array}$ & $\begin{array}{l}\text { Used as a disinfectant for wounds } \\
\text { and an anti-inflammatory agent and } \\
\text { also against arthritis. }\end{array}$ & NM & $\begin{array}{l}\text { Kashmir } \\
\text { Himalayas }\end{array}$ & $\begin{array}{l}\text { Pala et al., } \\
2013\end{array}$ \\
\hline 72. & \multirow{2}{*}{$\begin{array}{l}\text { Ganoderma } \\
\text { applanatum } \\
\text { (Pers.) Pat. }\end{array}$} & Heand & $\begin{array}{l}\text { Local people of } \\
\text { Jammu and Kashmir }\end{array}$ & $\begin{array}{l}\text { Used for the patients with weak } \\
\text { immune systems, against cough and } \\
\text { common cold. }\end{array}$ & $\begin{array}{l}\text { Local herbalists use it along with } \\
\text { honey and Kaloongi. }\end{array}$ & $\begin{array}{l}\text { Northern } \\
\text { Districts of } \\
\text { Jammu and } \\
\text { Kashmir. }\end{array}$ & $\begin{array}{l}\text { Malik et al. } \\
2017\end{array}$ \\
\hline 73. & & Soorkul lashe & $\begin{array}{l}\text { Local people of } \\
\text { Kashmir }\end{array}$ & $\begin{array}{l}\text { Used the belief that it reduces the } \\
\text { chances of disease. }\end{array}$ & $\begin{array}{l}\text { The powder obtained from } \\
\text { the dry fruiting body is added } \\
\text { to vegetables in very small } \\
\text { quantities during cooking. }\end{array}$ & $\begin{array}{l}\text { Kashmir } \\
\text { Himalayas }\end{array}$ & $\begin{array}{l}\text { Pala et al., } \\
2013\end{array}$ \\
\hline 74. & $\begin{array}{l}\text { Geopora } \\
\text { sumneriana } \\
\text { (Cooke) M. Torre }\end{array}$ & Shage Kan & $\begin{array}{l}\text { Local people of } \\
\text { Jammu and Kashmir. }\end{array}$ & $\begin{array}{l}\text { Used as stimulant and } \\
\text { tonic, anticold and also as } \\
\text { immunomodulator. }\end{array}$ & $\begin{array}{l}\text { Its powder is taken along with } \\
\text { milk and sugar before sleep. }\end{array}$ & $\begin{array}{l}\text { Northern } \\
\text { Districts of } \\
\text { Jammu and } \\
\text { Kashmir. }\end{array}$ & $\begin{array}{l}\text { Malik et al. } \\
2017\end{array}$ \\
\hline 75. & $\begin{array}{l}\text { Helvella } \\
\text { acetabulum (L.) } \\
\text { Quel }\end{array}$ & Heand & $\begin{array}{l}\text { Local people of } \\
\text { Jammu and Kashmir. }\end{array}$ & Used as chronic cough. & NM & $\begin{array}{l}\text { Northern } \\
\text { Districts of } \\
\text { Jammu and } \\
\text { Kashmir. }\end{array}$ & $\begin{array}{l}\text { Malik et al. } \\
2017\end{array}$ \\
\hline 76. & $\begin{array}{l}\text { Helvella crispa } \\
\text { Bull }\end{array}$ & Batta Heand & $\begin{array}{l}\text { Local people of } \\
\text { Jammu and Kashmir. }\end{array}$ & $\begin{array}{l}\text { For treatment of asthma, cough, } \\
\text { releases intestinal inflammation and } \\
\text { mouth ulceration. }\end{array}$ & $\begin{array}{l}\text { Powdered form is taken with } \\
\text { milk to get relief from intestinal } \\
\text { inflammation. }\end{array}$ & $\begin{array}{l}\text { Northern } \\
\text { Districts of } \\
\text { Jammu and } \\
\text { Kashmir. }\end{array}$ & $\begin{array}{l}\text { Malik et al. } \\
2017\end{array}$ \\
\hline
\end{tabular}




\begin{tabular}{|c|c|c|c|c|c|c|c|}
\hline Sl.No. & $\begin{array}{l}\text { Mushroom } \\
\text { names }\end{array}$ & $\begin{array}{l}\text { Vernacular } \\
\text { names }\end{array}$ & $\begin{array}{l}\text { Name of tribe or } \\
\text { local people }\end{array}$ & Used as & Preparations for use & $\begin{array}{l}\text { Reported from } \\
\text { India }\end{array}$ & References \\
\hline 77. & \multirow{3}{*}{$\begin{array}{l}\text { Hericium } \\
\text { coralloides } \\
\text { (Scop.) Pers. } \\
\text { Lactarius } \\
\text { deliciosus (L.: Fr.) } \\
\text { Gray }\end{array}$} & Kukur Panj & $\begin{array}{l}\text { Local people of } \\
\text { Jammu and Kashmir. }\end{array}$ & $\begin{array}{l}\text { Used for anticold, brain tonic, } \\
\text { suggested food for diabetic patients } \\
\text { also. }\end{array}$ & NM & $\begin{array}{l}\text { Northern } \\
\text { Districts of } \\
\text { Jammu and } \\
\text { Kashmir. }\end{array}$ & $\begin{array}{l}\text { Malik et al., } \\
2017\end{array}$ \\
\hline 78. & & Yaad gab & $\begin{array}{l}\text { Local people of } \\
\text { Kashmir }\end{array}$ & $\begin{array}{l}\text { It is widely consumed with the } \\
\text { belief that it lowers the risk of } \\
\text { cancer, heart disease and also } \\
\text { recommends macro fungus for } \\
\text { patients with hypertension. }\end{array}$ & NM & $\begin{array}{l}\text { Kashmir } \\
\text { Himalayas }\end{array}$ & $\begin{array}{l}\text { Pala et al., } \\
2013\end{array}$ \\
\hline 79. & & Heand & $\begin{array}{l}\text { Local people of } \\
\text { Jammu and Kashmir }\end{array}$ & $\begin{array}{l}\text { Used as sex stimulants and also } \\
\text { useful for gastric irritation and } \\
\text { heart burns. }\end{array}$ & $\begin{array}{l}\text { Used along with herbal } \\
\text { medicines (Sharbat bazoori } \\
\text { motadil or Saikanjabeen lee } \\
\text { moon). }\end{array}$ & $\begin{array}{l}\text { Northern } \\
\text { Districts of } \\
\text { Jammu and } \\
\text { Northern } \\
\text { Districts of } \\
\text { Jammu and } \\
\text { Kashmir. }\end{array}$ & $\begin{array}{l}\text { Malik et al., } \\
2017\end{array}$ \\
\hline 80. & \multirow{3}{*}{$\begin{array}{l}\text { Lentinus tigrinus } \\
\text { (Bull.) Fr. }\end{array}$} & Kater & $\begin{array}{l}\text { Local people of } \\
\text { Kashmir }\end{array}$ & $\begin{array}{l}\text { It is believed to enhance the } \\
\text { defensive mechanisms of the body, } \\
\text { also used for the treatment of } \\
\text { frostbite. }\end{array}$ & $\begin{array}{l}\text { Dried fruiting body is crushed to } \\
\text { form powder. }\end{array}$ & $\begin{array}{l}\text { Kashmir } \\
\text { Himalayas }\end{array}$ & $\begin{array}{l}\text { Pala et al., } \\
2013\end{array}$ \\
\hline 81. & & Veeri Heand & $\begin{array}{l}\text { Local people of } \\
\text { Jammu and Kashmir }\end{array}$ & $\begin{array}{l}\text { Widely used as food, brain tonic } \\
\text { and against anger, dry cough, } \\
\text { asthma and enhances digestion. }\end{array}$ & $\begin{array}{l}\text { Powder of the dried mushroom } \\
\text { is taken in hot water in case of } \\
\text { dry cough and asthma. It is taken } \\
\text { raw to cure wet cough. Raw } \\
\text { slices along with lemon juice are } \\
\text { advised in case of indigestion. }\end{array}$ & $\begin{array}{l}\text { Northern } \\
\text { Districts of } \\
\text { Jammu and } \\
\text { Kashmir. }\end{array}$ & $\begin{array}{l}\text { Malik et al., } \\
2017\end{array}$ \\
\hline 82. & & Vire haddur & $\begin{array}{l}\text { Local people of } \\
\text { Kashmir }\end{array}$ & Used for diabetic patient. & $\mathrm{NM}$ & $\begin{array}{l}\text { Kashmir } \\
\text { Himalayas }\end{array}$ & $\begin{array}{l}\text { Pala et al., } \\
2013\end{array}$ \\
\hline 83. & $\begin{array}{l}\text { Morchella conica } \\
\text { (Pers.) Fr. }\end{array}$ & Zande guech & $\begin{array}{l}\text { Local people of } \\
\text { Jammu and Kashmir }\end{array}$ & $\begin{array}{l}\text { Used for heart ailments, brain tonic, } \\
\text { arthritis general weakness and sex } \\
\text { stimulant. }\end{array}$ & $\begin{array}{l}\text { If taken along with dimagheen } \\
\text { (herbal medicine), pomegranate } \\
\text { and honey then its efficacy } \\
\text { enhances the mentioned } \\
\text { ailments. }\end{array}$ & $\begin{array}{l}\text { Northern } \\
\text { Districts of } \\
\text { Jammu and } \\
\text { Kashmir. }\end{array}$ & $\begin{array}{l}\text { Malik et al., } \\
2017\end{array}$ \\
\hline 84. & \multirow{2}{*}{$\begin{array}{l}\text { Morchella } \\
\text { esculenta Fr. }\end{array}$} & Batta Guchi & $\begin{array}{l}\text { Local people of } \\
\text { Jammu and Kashmir }\end{array}$ & $\begin{array}{l}\text { Used for heart ailments, general } \\
\text { weakness, brain tonic, arthritis. }\end{array}$ & NM & $\begin{array}{l}\text { Northern } \\
\text { Districts of } \\
\text { Jammu and } \\
\text { Kashmir. }\end{array}$ & $\begin{array}{l}\text { Malik et al., } \\
2017\end{array}$ \\
\hline 85. & & $\begin{array}{l}\text { Kann guch, } \\
\text { Guuch }\end{array}$ & $\begin{array}{l}\text { Local people of } \\
\text { Kashmir }\end{array}$ & $\begin{array}{l}\text { Used as sexual weakness } \\
\text { (aphrodisiac) of men. }\end{array}$ & $\begin{array}{l}\text { Paste of the dried fruiting } \\
\text { body is blended with a glass of } \\
\text { lukewarm milk. }\end{array}$ & $\begin{array}{l}\text { Kashmir } \\
\text { Himalayas }\end{array}$ & $\begin{array}{l}\text { Pala et al., } \\
2013\end{array}$ \\
\hline 86. & $\begin{array}{l}\text { Morchella hybrid } \\
\text { (Fr.) Cetto }\end{array}$ & Saaz guech & $\begin{array}{l}\text { Local people of } \\
\text { Jammu and Kashmir }\end{array}$ & $\begin{array}{l}\text { Used for heart ailments, general } \\
\text { weakness, brain tonic, arthritis. }\end{array}$ & NM & $\begin{array}{l}\text { Northern } \\
\text { Districts of } \\
\text { Jammu and } \\
\text { Kashmir. }\end{array}$ & $\begin{array}{l}\text { Malik et al., } \\
2017\end{array}$ \\
\hline 87. & \multirow[t]{2}{*}{$\begin{array}{l}\text { Morchella } \\
\text { vulgaris (Pers.) } \\
\text { Boud. }\end{array}$} & $\begin{array}{l}\text { Kreuhn guech/ } \\
\text { Dumguch }\end{array}$ & $\begin{array}{l}\text { Local people of } \\
\text { Jammu and Kashmir }\end{array}$ & $\begin{array}{l}\text { Used for heart ailments, general } \\
\text { weakness, brain tonic, arthritis. }\end{array}$ & NM & $\begin{array}{l}\text { Northern } \\
\text { Districts of } \\
\text { Jammu and } \\
\text { Kashmir. }\end{array}$ & $\begin{array}{l}\text { Malik et al., } \\
2017\end{array}$ \\
\hline 88. & & $\begin{array}{l}\text { Kann guch, } \\
\text { Guuch }\end{array}$ & $\begin{array}{l}\text { Local people of } \\
\text { Kashmir }\end{array}$ & $\begin{array}{l}\text { Used it for treatment of respiratory } \\
\text { problems and as an aphrodisiac. }\end{array}$ & NM & $\begin{array}{l}\text { Kashmir } \\
\text { Himalayas }\end{array}$ & $\begin{array}{l}\text { Pala et al., } \\
2013\end{array}$ \\
\hline 89. & \multirow{2}{*}{$\begin{array}{l}\text { Phallus impudicus } \\
\text { L. }\end{array}$} & Paane laet & $\begin{array}{l}\text { Local people of } \\
\text { Jammu and Kashmir }\end{array}$ & Used for healing of wounds. & NM & $\begin{array}{l}\text { Northern } \\
\text { Districts of } \\
\text { Jammu and } \\
\text { Kashmir. }\end{array}$ & $\begin{array}{l}\text { Malik et al., } \\
2017\end{array}$ \\
\hline 90. & & Sawan guch & Gujar & $\begin{array}{l}\text { The gelatinous material inside the } \\
\text { egg covering of mushroom is used } \\
\text { for healing wounds and also for } \\
\text { burns. }\end{array}$ & $\mathrm{NM}$ & $\begin{array}{l}\text { Kashmir } \\
\text { Himalayas }\end{array}$ & $\begin{array}{l}\text { Pala et al., } \\
2013\end{array}$ \\
\hline
\end{tabular}




\begin{tabular}{|c|c|c|c|c|c|c|c|}
\hline Sl.No. & $\begin{array}{l}\text { Mushroom } \\
\text { names }\end{array}$ & $\begin{array}{l}\text { Vernacular } \\
\text { names }\end{array}$ & $\begin{array}{l}\text { Name of tribe or } \\
\text { local people }\end{array}$ & Used as & Preparations for use & $\begin{array}{l}\text { Reported from } \\
\text { India }\end{array}$ & References \\
\hline 91. & \multirow{4}{*}{$\begin{array}{l}\text { Pleurotus } \\
\text { ostreatus } \\
\text { (Jacq.:Fr.) } \\
\text { P.Kumm. }\end{array}$} & Kuel Heand & $\begin{array}{l}\text { Local people of } \\
\text { Jammu and Kashmir }\end{array}$ & $\begin{array}{l}\text { Used as tonic against weakness as a } \\
\text { result of prolonged illness. }\end{array}$ & NM & $\begin{array}{l}\text { Northern } \\
\text { Districts of } \\
\text { Jammu and } \\
\text { Kashmir }\end{array}$ & $\begin{array}{l}\text { Malik et al., } \\
2017\end{array}$ \\
\hline 92. & & $\mathrm{NM}$ & $\begin{array}{l}\text { Santal, Kolha, } \\
\text { Munda, Khadia, } \\
\text { Bhumija, Bhuyan, } \\
\text { Bathudi, Kudumi, Ho, } \\
\text { Mankdias }\end{array}$ & $\begin{array}{l}\text { Used for the treatments of asthma } \\
\text { and lowering high blood pressure. }\end{array}$ & NM & $\begin{array}{l}\text { Similipal } \\
\text { biosphere } \\
\text { reserve, Odisha }\end{array}$ & $\begin{array}{l}\text { Sachan et al., } \\
2013\end{array}$ \\
\hline 93. & & Lutur Onth & $\begin{array}{l}\text { Santal, Lodha, and } \\
\text { Dhangar }\end{array}$ & $\begin{array}{l}\text { Yellow-colored spores are used } \\
\text { as medicine for the treatment of } \\
\text { wounds. }\end{array}$ & NM & $\begin{array}{l}\text { Eastern Lateritic } \\
\text { Part of India }\end{array}$ & $\begin{array}{l}\text { Manna et al., } \\
2014\end{array}$ \\
\hline 94. & & $\begin{array}{l}\text { Khodmand } \\
\text { hadur }\end{array}$ & $\begin{array}{l}\text { Local people of } \\
\text { Kashmir }\end{array}$ & $\begin{array}{l}\text { Used for the treatment of } \\
\text { hypertension, diabetes, jaundice, } \\
\text { and asthma. It is also believed to } \\
\text { reduce the chances of tumour. }\end{array}$ & NM & $\begin{array}{l}\text { Kashmir } \\
\text { Himalayas }\end{array}$ & $\begin{array}{l}\text { Pala et al., } \\
2013\end{array}$ \\
\hline 95. & $\begin{array}{l}\text { Ramaria stricta } \\
\text { (Pers.) Quel. }\end{array}$ & Rai Saad & $\begin{array}{l}\text { Local people of } \\
\text { Jammu and Kashmir }\end{array}$ & $\begin{array}{l}\text { Used for the treatments of asthma, } \\
\text { other respiratory ailments, blood } \\
\text { purifier and enhances skin colour } \\
\text { and eye ailments. }\end{array}$ & $\begin{array}{l}\text { Its powder along with herbal } \\
\text { medicines Stumble tayeb and } \\
\text { Shahtara purifies blood. In } \\
\text { winters its powder along with } \\
\text { pepper and clove is useful } \\
\text { against lung inflammation. }\end{array}$ & $\begin{array}{l}\text { Northern } \\
\text { Districts of } \\
\text { Jammu and } \\
\text { Kashmir }\end{array}$ & $\begin{array}{l}\text { Malik et al., } \\
2017\end{array}$ \\
\hline 96. & $\begin{array}{l}\text { Rhizopogon } \\
\text { villosulus Zeller }\end{array}$ & $\begin{array}{l}\text { Deodar } \\
\text { Mungrae }\end{array}$ & $\begin{array}{l}\text { Local people of } \\
\text { Jammu and Kashmir }\end{array}$ & $\begin{array}{l}\text { Used against kidney stones, urinary } \\
\text { tract infections, and also used to } \\
\text { cure fatty liver and asthma. }\end{array}$ & $\begin{array}{l}\text { In case of kidney stones its } \\
\text { powder along with herbal extract } \\
\text { of Aaloo Baloo and Sang i } \\
\text { sarmahi is used. In combine with } \\
\text { Zoofa an ayurvedic medicine } \\
\text { cures fatty liver and asthma. }\end{array}$ & $\begin{array}{l}\text { Northern } \\
\text { Districts of } \\
\text { Jammu and } \\
\text { Kashmir }\end{array}$ & $\begin{array}{l}\text { Malik et al., } \\
2017\end{array}$ \\
\hline 97. & $\begin{array}{l}\text { Rhizopogon } \\
\text { roseolus Corda }\end{array}$ & $\begin{array}{l}\text { Madaan } \\
\text { Mungrae }\end{array}$ & $\begin{array}{l}\text { Local people of } \\
\text { Jammu and Kashmir }\end{array}$ & $\begin{array}{l}\text { Used for the treatment of urinary } \\
\text { tract infections. }\end{array}$ & NM & $\begin{array}{l}\text { Northern } \\
\text { Districts of } \\
\text { Jammu and } \\
\text { Kashmir }\end{array}$ & $\begin{array}{l}\text { Malik et al., } \\
2017\end{array}$ \\
\hline 98. & $\begin{array}{l}\text { Sparassis crispa } \\
\text { Wulfen }\end{array}$ & Kawa khour & $\begin{array}{l}\text { Local people of } \\
\text { Jammu and Kashmir }\end{array}$ & $\begin{array}{l}\text { Used as general tonic, blood } \\
\text { purifier, anticold, against memory } \\
\text { loss, depression, anger and chest } \\
\text { pains. }\end{array}$ & $\begin{array}{l}\text { Local hakims use its powder } \\
\text { along with Aaloo Baloo (herbal } \\
\text { medicine) in the ratio of } 1: 5 \text {. }\end{array}$ & $\begin{array}{l}\text { Northern } \\
\text { Districts of } \\
\text { Jammu and } \\
\text { Kashmir }\end{array}$ & $\begin{array}{l}\text { Malik et al., } \\
2017\end{array}$ \\
\hline 99. & $\begin{array}{l}\text { Sparassis } \\
\text { spathulata } \\
\text { (Schwein.) Fr }\end{array}$ & $\begin{array}{l}\text { Raisaad/ } \\
\text { Kawkhour }\end{array}$ & $\begin{array}{l}\text { Local people of } \\
\text { Jammu and Kashmir }\end{array}$ & $\begin{array}{l}\text { Mainly used as General tonic and } \\
\text { anticold, blood purifier. }\end{array}$ & Cooked along with milk. & $\begin{array}{l}\text { Northern } \\
\text { Districts of } \\
\text { Jammu and } \\
\text { Kashmir }\end{array}$ & $\begin{array}{l}\text { Malik et al., } \\
2017\end{array}$ \\
\hline 100. & $\begin{array}{l}\text { Trametes } \\
\text { versicolor }(\mathrm{L} .) \\
\text { Lloyd. H }\end{array}$ & Heand & $\begin{array}{l}\text { Local people of } \\
\text { Jammu and Kashmir }\end{array}$ & $\begin{array}{l}\text { Effective against skin diseases such } \\
\text { as rashes, itching, dryness, and } \\
\text { healing of wounds. }\end{array}$ & Used with butter or oil. & $\begin{array}{l}\text { Northern } \\
\text { Districts of } \\
\text { Jammu and } \\
\text { Kashmir }\end{array}$ & $\begin{array}{l}\text { Malik et al., } \\
2017\end{array}$ \\
\hline 101. & & $\begin{array}{l}\text { Mukhmal } \\
\text { gunde }\end{array}$ & $\begin{array}{l}\text { Local people of } \\
\text { Kashmir }\end{array}$ & Used against dermatitis. & NM & $\begin{array}{l}\text { Kashmir } \\
\text { Himalayas }\end{array}$ & $\begin{array}{l}\text { Pala et al., } \\
2013\end{array}$ \\
\hline 102. & $\begin{array}{l}\text { Termitomyces } \\
\text { mammiformies }\end{array}$ & NM & Mokokchung & $\begin{array}{l}\text { Used for the treatment of } \\
\text { abdominal discomfort, cough and } \\
\text { whooping cough. }\end{array}$ & NM & $\begin{array}{l}\text { Nagaland, North } \\
\text { East India }\end{array}$ & $\begin{array}{l}\text { Kumar et al., } \\
2014\end{array}$ \\
\hline 103. & $\begin{array}{l}\text { Auricularia } \\
\text { auricular } \\
\text { (Hooker) }\end{array}$ & NM & Mokokchung & Used as medicine. & NM & $\begin{array}{l}\text { Nagaland, North } \\
\text { East India }\end{array}$ & $\begin{array}{l}\text { Kumar et al., } \\
2014\end{array}$ \\
\hline 104. & $\begin{array}{l}\text { Lentinula edodes } \\
\text { (Berk.) Pegler }\end{array}$ & NM & $\begin{array}{l}\text { Local people of } \\
\text { Assam. }\end{array}$ & $\begin{array}{l}\text { Used to increase general strength } \\
\text { and immunity. }\end{array}$ & NM & $\begin{array}{l}\text { Kamrup District, } \\
\text { Assam. }\end{array}$ & Devi, 2017 \\
\hline 105. & Russula delica Fr. & NM & $\begin{array}{l}\text { Santal, Kolha, } \\
\text { Munda, Khadia, } \\
\text { Bhumija, Bhuyan, } \\
\text { Bathudi, Kudumi, Ho, } \\
\text { Mankdias }\end{array}$ & $\begin{array}{l}\text { Used in malnutrition, weakness and } \\
\text { nutritional disorder. }\end{array}$ & NM & $\begin{array}{l}\text { Similipal } \\
\text { biosphere } \\
\text { reserve, Odisha }\end{array}$ & $\begin{array}{l}\text { Sachan et al., } \\
2013\end{array}$ \\
\hline 106. & $\begin{array}{l}\text { Termitomyces } \\
\text { eurrhizus (Berk.) } \\
\text { R. Heim }\end{array}$ & NM & $\begin{array}{l}\text { Santal, Kolha, } \\
\text { Munda, Khadia, } \\
\text { Bhumija, Bhuyan, } \\
\text { Bathudi, Kudumi, Ho, } \\
\text { Mankdias }\end{array}$ & $\begin{array}{l}\text { Used for the treatments of } \\
\text { rheumatism, diarrhoea, lowering } \\
\text { high blood pressure. }\end{array}$ & NM & $\begin{array}{l}\text { Similipal } \\
\text { biosphere } \\
\text { reserve, Odisha }\end{array}$ & $\begin{array}{l}\text { Sachan et al., } \\
2013\end{array}$ \\
\hline
\end{tabular}




\begin{tabular}{|c|c|c|c|c|c|c|c|}
\hline Sl.No. & $\begin{array}{l}\text { Mushroom } \\
\text { names }\end{array}$ & $\begin{array}{l}\text { Vernacular } \\
\text { names }\end{array}$ & $\begin{array}{l}\text { Name of tribe or } \\
\text { local people }\end{array}$ & Used as & Preparations for use & $\begin{array}{l}\text { Reported from } \\
\text { India }\end{array}$ & References \\
\hline 107. & Termitomyces sp. & NM & $\begin{array}{l}\text { Santal, Kolha, } \\
\text { Munda, Khadia, } \\
\text { Bhumija, Bhuyan, } \\
\text { Bathudi, Kudumi, Ho, } \\
\text { Mankdias }\end{array}$ & $\begin{array}{l}\text { Used for the treatments of } \\
\text { rheumatism, diarrhoea, lowering } \\
\text { high blood pressure. }\end{array}$ & NM & $\begin{array}{l}\text { Similipal } \\
\text { biosphere } \\
\text { reserve, Odisha }\end{array}$ & $\begin{array}{l}\text { Sachan et al., } \\
2013\end{array}$ \\
\hline 108. & $\begin{array}{l}\text { Agaricus } \\
\text { silvaticus } \text { Schaeff. }\end{array}$ & NM & $\begin{array}{l}\text { Santal, Kolha, } \\
\text { Munda, Khadia, } \\
\text { Bhumija, Bhuyan, } \\
\text { Bathudi, Kudumi, Ho, } \\
\text { Mankdias }\end{array}$ & Used in high fever. & NM & $\begin{array}{l}\text { Similipal } \\
\text { biosphere } \\
\text { reserve, Odisha }\end{array}$ & $\begin{array}{l}\text { Sachan et al., } \\
2013\end{array}$ \\
\hline 109. & $\begin{array}{l}\text { Volvorella } \\
\text { volvacea } \\
\text { (Bul.) Singer }\end{array}$ & NM & $\begin{array}{l}\text { Santal, Kolha, } \\
\text { Munda, Khadia, } \\
\text { Bhumija, Bhuyan, } \\
\text { Bathudi, Kudumi, Ho, } \\
\text { Mankdias }\end{array}$ & $\begin{array}{l}\text { Used for lowering high blood } \\
\text { pressure. }\end{array}$ & NM & $\begin{array}{l}\text { Similipal } \\
\text { biosphere } \\
\text { reserve, Odisha }\end{array}$ & $\begin{array}{l}\text { Sachan et al., } \\
2013\end{array}$ \\
\hline 110. & $\begin{array}{l}\text { Lentinus sajor- } \\
\text { caju (Fr.) Fr. }\end{array}$ & NM & $\begin{array}{l}\text { Santal, Kolha, } \\
\text { Munda, Khadia, } \\
\text { Bhumija, Bhuyan, } \\
\text { Bathudi, Kudumi, Ho, } \\
\text { Mankdias }\end{array}$ & Used in cold and cough & NM & $\begin{array}{l}\text { Similipal } \\
\text { biosphere } \\
\text { reserve, Odisha }\end{array}$ & $\begin{array}{l}\text { Sachan et al., } \\
2013\end{array}$ \\
\hline 111. & Lycoperdon sp. & NM & $\begin{array}{l}\text { Santal, Kolha, } \\
\text { Munda, Khadia, } \\
\text { Bhumija, Bhuyan, } \\
\text { Bathudi, Kudumi, Ho, } \\
\text { Mankdias }\end{array}$ & Used to cure wound. & NM & $\begin{array}{l}\text { Similipal } \\
\text { biosphere } \\
\text { reserve, Odisha }\end{array}$ & $\begin{array}{l}\text { Sachan et al., } \\
2013\end{array}$ \\
\hline 112. & Pleurotus sp. & $\mathrm{NM}$ & $\begin{array}{l}\text { Santal, Kolha, } \\
\text { Munda, Khadia, } \\
\text { Bhumija, Bhuyan, } \\
\text { Bathudi, Kudumi, Ho, } \\
\text { Mankdias }\end{array}$ & $\begin{array}{l}\text { Used for the treatments of asthma } \\
\text { and lowering high blood pressure }\end{array}$ & NM & $\begin{array}{l}\text { Similipal } \\
\text { biosphere } \\
\text { reserve, Odisha }\end{array}$ & $\begin{array}{l}\text { Sachan et al., } \\
2013\end{array}$ \\
\hline 113. & Xylaria sp. & $\begin{array}{l}\text { More-pankh } \\
\text { (peacock } \\
\text { feather) }\end{array}$ & Ghanashia and Rabaris & $\begin{array}{l}\text { Used for the treatments of } \\
\text { pneumonia, constipation, eczema, } \\
\text { and pneumonia like symptoms. }\end{array}$ & $\begin{array}{l}\text { Around } 5 \text { to } 6 \text { entire fruiting } \\
\text { bodies are triturated fresh with } \\
\text { water for several times to form } \\
\text { a uniform paste. The paste is } \\
\text { consumed orally for pneumonia } \\
\text { or constipation. Generally for } \\
\text { pneumonia } 1 \text { tablespoon is } \\
\text { administered for } 3-5 \text { times on } \\
\text { different days. } \\
\text { For sever constipation same } \\
\text { dose is given after dinner for } 2 \\
\text { to } 3 \text { days. The paste is applied } \\
\text { topically for eczema } \\
\text { In case of eczema the affected } \\
\text { area is covered with the paste } \\
\text { and bandaged. }\end{array}$ & Gujarat & $\begin{array}{l}\text { Lahiri et al., } \\
2010\end{array}$ \\
\hline 114. & Phallus sp. & Datto (button) & Ghanashia and Rabaris & $\begin{array}{l}\text { Used in wound, skin infections, } \\
\text { boils, or lesions. }\end{array}$ & $\begin{array}{l}\text { The cap is crushed in mortar } \\
\text { with water and applied } \\
\text { on affected area but is not } \\
\text { bandaged. } \\
\text { The application is repeated twice } \\
\text { everyday for } 3 \text { days or longer } \\
\text { if needed. }\end{array}$ & Gujarat & $\begin{array}{l}\text { Lahiri et al., } \\
2010\end{array}$ \\
\hline 115. & $\begin{array}{l}\text { Lepiota cristata } \\
\text { (Bolton) P.Kumm. }\end{array}$ & $\begin{array}{l}\text { Chatri } \\
\text { (umbrella) }\end{array}$ & Ghanashia and Rabaris & $\begin{array}{l}\text { Used for the boils to speed its } \\
\text { maturation and the regression. }\end{array}$ & $\begin{array}{l}\text { The entire mushroom is crushed } \\
\text { in mortar and applied. One or } \\
\text { two applications are sufficient } \\
\text { to treat. }\end{array}$ & Gujarat & $\begin{array}{l}\text { Lahiri et al., } \\
2010\end{array}$ \\
\hline 116. & Scleroderma sp. & Dado (ball) & Ghanashia and Rabaris & Used for physical afflicted wounds. & $\begin{array}{l}\text { The gleba is triturated in water } \\
\text { and applied over wounds and } \\
\text { bandaged. The same is repeated } \\
\text { for } 2 \text { to } 3 \text { times or till total } \\
\text { recovery is confirmed. }\end{array}$ & Gujarat & $\begin{array}{l}\text { Lahiri et al., } \\
2010\end{array}$ \\
\hline
\end{tabular}




\begin{tabular}{|c|c|c|c|c|c|c|c|}
\hline SI.No. & $\begin{array}{l}\text { Mushroom } \\
\text { names }\end{array}$ & $\begin{array}{l}\text { Vernacular } \\
\text { names }\end{array}$ & $\begin{array}{l}\text { Name of tribe or } \\
\text { local people }\end{array}$ & Used as & Preparations for use & $\begin{array}{l}\text { Reported from } \\
\text { India }\end{array}$ & References \\
\hline 117. & $\begin{array}{l}\text { Termitomyces } \\
\text { tyleranus Otieno }\end{array}$ & Adim & Dangi people & $\begin{array}{l}\text { It is used to treat on general } \\
\text { protection against diseases or with } \\
\text { special reference to chicken pox. }\end{array}$ & $\begin{array}{l}\text { It is dried and used for } \\
\text { prophylaxis throughout the } \\
\text { year for general protection } \\
\text { against diseases or with special } \\
\text { reference to chicken pox. Also } \\
\text { burned and inhaled to speed } \\
\text { up the occurrence, maturation } \\
\text { and scaling of chicken pox. } \\
\text { Cooked or raw mushrooms are } \\
\text { consumed orally. }\end{array}$ & Gujarat & $\begin{array}{l}\text { Lahiri et al., } \\
2010\end{array}$ \\
\hline 118. & $\begin{array}{l}\text { Phellinus } \\
\text { durissimus } \\
\text { (Lloyd) A. Roy }\end{array}$ & $\begin{array}{l}\text { Galpacodio } \\
\text { (addressing } \\
\text { mumps) }\end{array}$ & Dangi people & $\begin{array}{l}\text { It is used to cure mumps affected } \\
\text { edema and simple edema. }\end{array}$ & $\begin{array}{l}\text { Generally it is triturated in water } \\
\text { and applied on mumps affected } \\
\text { edema, fruiting body heated } \\
\text { and padded on simple edema or } \\
\text { inflammation. }\end{array}$ & Gujarat & $\begin{array}{l}\text { Lahiri et al., } \\
2010\end{array}$ \\
\hline 119. & Bovista sp. & Bhupid & Dangi people & $\begin{array}{l}\text { The spore mass used as topical } \\
\text { application on bruised skin } \\
\text { infections directly till recovery }\end{array}$ & NM & Gujarat & $\begin{array}{l}\text { Lahiri et al., } \\
2010\end{array}$ \\
\hline 120. & Pleurotus sp. & NM & Dangi people & $\begin{array}{l}\text { It is used to impart strengthening } \\
\text { effect. }\end{array}$ & Consumed orally after cooking. & Gujarat & $\begin{array}{l}\text { Lahiri et al., } \\
2010\end{array}$ \\
\hline 121. & Lentinus sp. & $\begin{array}{l}\text { Vansarta } \\
\text { (growing on } \\
\text { bamboo }\end{array}$ & Dangi people & $\begin{array}{l}\text { Used for better health, specifically } \\
\text { during convalescence. }\end{array}$ & $\begin{array}{l}\text { It is stored through the year as } \\
\text { powder and added in soups for } \\
\text { better health. }\end{array}$ & Gujarat & $\begin{array}{l}\text { Lahiri et al., } \\
2010\end{array}$ \\
\hline 122. & $\begin{array}{l}\text { Amanita muscaria } \\
\text { var. formosa (Pers. } \\
\text { : Fr.) Chambre }\end{array}$ & Zahare heddu & $\begin{array}{l}\text { Local people of } \\
\text { Kashmir }\end{array}$ & Used for rheumatoid arthritis. & $\begin{array}{l}\text { Preparations from dry powder of } \\
\text { mushroom are used. }\end{array}$ & $\begin{array}{l}\text { Kashmir } \\
\text { Himalayas }\end{array}$ & $\begin{array}{l}\text { Pala et al., } \\
2013\end{array}$ \\
\hline 123. & $\begin{array}{l}\text { Coprinus } \\
\text { atramentarius } \\
\text { (Bull. : Fr.) Fr }\end{array}$ & Sakerbub & $\begin{array}{l}\text { Local people of } \\
\text { Kashmir }\end{array}$ & $\begin{array}{l}\text { Its acid formulations are used in } \\
\text { the treatment of kidney problems, } \\
\text { pus cells in urine, and removal of } \\
\text { gall stones. }\end{array}$ & NM & $\begin{array}{l}\text { Kashmir } \\
\text { Himalayas }\end{array}$ & $\begin{array}{l}\text { Pala et al., } \\
2013\end{array}$ \\
\hline 124. & $\begin{array}{l}\text { Coprinus } \\
\text { micaceus (Bull.: } \\
\text { Fr.)Fr. }\end{array}$ & Hoon haddur & $\begin{array}{l}\text { Local people of } \\
\text { Kashmir }\end{array}$ & Used to treat skin infections. & NM & $\begin{array}{l}\text { Kashmir } \\
\text { Himalayas }\end{array}$ & $\begin{array}{l}\text { Pala et al., } \\
2013\end{array}$ \\
\hline 125. & $\begin{array}{l}\text { Fomitopsis } \\
\text { pinicola }(\mathrm{Sw} . \text { :Fr.) } \\
\text { P. Krast }\end{array}$ & Yaade lashe & $\begin{array}{l}\text { Local people of } \\
\text { Kashmir }\end{array}$ & $\begin{array}{l}\text { Used to stop bleeding of minor } \\
\text { physical injuries and rheumatoid } \\
\text { arthritis. }\end{array}$ & $\begin{array}{l}\text { Dried fruitbody of the } \\
\text { mushroom is crushed and turned } \\
\text { into powdered form and used in } \\
\text { case of injuries.From that extract } \\
\text { is prepared and used in case of } \\
\text { arthritis. }\end{array}$ & $\begin{array}{l}\text { Kashmir } \\
\text { Himalayas }\end{array}$ & $\begin{array}{l}\text { Pala et al., } \\
2013\end{array}$ \\
\hline 126. & $\begin{array}{l}\text { Gomphus } \\
\text { floccosus } \\
\text { (Schwn.) Singer }\end{array}$ & Makhmle kanz & $\begin{array}{l}\text { Local people of } \\
\text { Kashmir }\end{array}$ & $\begin{array}{l}\text { Used externally for eczema and } \\
\text { athlete's foot disease. }\end{array}$ & $\begin{array}{l}\text { Paste is obtained from the } \\
\text { sporocarp and used to treat } \\
\text { eczema. Extract is used in case } \\
\text { of athlete's disease. }\end{array}$ & $\begin{array}{l}\text { Kashmir } \\
\text { Himalayas }\end{array}$ & $\begin{array}{l}\text { Pala et al., } \\
2013\end{array}$ \\
\hline 127. & $\begin{array}{l}\text { Gyromitra } \\
\text { sphaerospora } \\
\text { (Peck) Sacc. }\end{array}$ & Buji kan kuch & $\begin{array}{l}\text { Local people of } \\
\text { Kashmir }\end{array}$ & Used for the treatment of goiter. & NM & $\begin{array}{l}\text { Kashmir } \\
\text { Himalayas }\end{array}$ & $\begin{array}{l}\text { Pala et al., } \\
2013\end{array}$ \\
\hline 128. & $\begin{array}{l}\text { Hevella lacunose } \\
\text { Afzl.: Fr. }\end{array}$ & Shajikunl & $\begin{array}{l}\text { Local people of } \\
\text { Kashmir }\end{array}$ & Used to cure piles. & NM & $\begin{array}{l}\text { Kashmir } \\
\text { Himalayas }\end{array}$ & $\begin{array}{l}\text { Pala et al., } \\
2013\end{array}$ \\
\hline 129. & $\begin{array}{l}\text { Helvella macropus } \\
\text { (Pers.) P. Karst. }\end{array}$ & Kanpapri & $\begin{array}{l}\text { Local people of } \\
\text { Kashmir }\end{array}$ & $\begin{array}{l}\text { Used for the treatment of asthma by } \\
\text { the tribal people. }\end{array}$ & NM & $\begin{array}{l}\text { Kashmir } \\
\text { Himalayas }\end{array}$ & $\begin{array}{l}\text { Pala et al., } \\
2013\end{array}$ \\
\hline 130. & $\begin{array}{l}\text { Humaria } \\
\text { hemisphaerica } \\
\text { (Wigg.) Fuckel }\end{array}$ & Panz kan & $\begin{array}{l}\text { Local people of } \\
\text { Kashmir }\end{array}$ & $\begin{array}{l}\text { Used by the tribal for the treatment } \\
\text { of blisters on skin. }\end{array}$ & $\begin{array}{l}\text { Preparation is made in } \\
\text { combination with mustard oil } \\
\text { and fruiting body. }\end{array}$ & $\begin{array}{l}\text { Kashmir } \\
\text { Himalayas }\end{array}$ & $\begin{array}{l}\text { Pala et al., } \\
2013\end{array}$ \\
\hline 131. & $\begin{array}{l}\text { Inonotus hispidus } \\
\text { (Bull.) P.Karst. }\end{array}$ & Chunth Lashe & $\begin{array}{l}\text { Local people of } \\
\text { Kashmir }\end{array}$ & $\begin{array}{l}\text { Used externally as a disinfectant } \\
\text { and for the treatment of boils. }\end{array}$ & $\begin{array}{l}\text { Extract obtained from the } \\
\text { fruiting body is used. }\end{array}$ & $\begin{array}{l}\text { Kashmir } \\
\text { Himalayas }\end{array}$ & $\begin{array}{l}\text { Pala et al., } \\
2013\end{array}$ \\
\hline 132. & $\begin{array}{l}\text { Langarmania } \\
\text { gigantia (Batsch } \\
\text { ex Pers.) Rostk }\end{array}$ & Jangli Aaloo & $\begin{array}{l}\text { Local people of } \\
\text { Kashmir }\end{array}$ & Used to cure stomach ulcers. & NM & $\begin{array}{l}\text { Kashmir } \\
\text { Himalayas }\end{array}$ & $\begin{array}{l}\text { Pala et al., } \\
2013\end{array}$ \\
\hline 133. & $\begin{array}{l}\text { Panaeolus } \\
\text { sphinctrinus (Fr.) } \\
\text { Quél. }\end{array}$ & Guhi haddur & $\begin{array}{l}\text { Local people of } \\
\text { Kashmir }\end{array}$ & Used as an aesthetic agent. & $\begin{array}{l}\text { The paste of the sporocarp is } \\
\text { blended with a tea in small } \\
\text { concentrations. }\end{array}$ & $\begin{array}{l}\text { Kashmir } \\
\text { Himalayas }\end{array}$ & $\begin{array}{l}\text { Pala et al., } \\
2013\end{array}$ \\
\hline 134. & $\begin{array}{l}\text { Peziza repanda } \\
\text { Pers. }\end{array}$ & Bati kucch & Gujar & $\begin{array}{l}\text { It is used against diabetes, } \\
\text { constipation, and eczema. }\end{array}$ & NM & $\begin{array}{l}\text { Kashmir } \\
\text { Himalayas }\end{array}$ & $\begin{array}{l}\text { Pala et al., } \\
2013\end{array}$ \\
\hline 135. & $\begin{array}{l}\text { Ramaria formosa } \\
\text { (Pers.) Quél. }\end{array}$ & $\begin{array}{l}\text { Panz anguj or } \\
\text { Hapat paanji }\end{array}$ & $\begin{array}{l}\text { Local people of } \\
\text { Kashmir }\end{array}$ & $\begin{array}{l}\text { It is also recommended for cardiac } \\
\text { and diabetic patients by local tribes. }\end{array}$ & NM & $\begin{array}{l}\text { Kashmir } \\
\text { Himalayas }\end{array}$ & $\begin{array}{l}\text { Pala et al., } \\
2013\end{array}$ \\
\hline 136. & $\begin{array}{l}\text { Verpa bohemica } \\
\text { (Krombh.) Schrot. }\end{array}$ & Paankat & $\begin{array}{l}\text { Local people of } \\
\text { Kashmir }\end{array}$ & $\begin{array}{l}\text { It is used for the treatment of } \\
\text { urinary disorders. }\end{array}$ & NM & $\begin{array}{l}\text { Kashmir } \\
\text { Himalayas }\end{array}$ & $\begin{array}{l}\text { Pala et al., } \\
2013\end{array}$ \\
\hline
\end{tabular}


review work, we have found that there are almost 18 reports about the traditional use of wild mushrooms as medicine from 14 states, namely, Uttarakhand, Gujarat, Madhya Pradesh, Assam, Nagaland, Sikkim, Jammu, Kashmir, Odisha, Tamilnadu, West Bengal, Bihar, Chhattisgarh and Jharkhand of India. This documentation suggested that, at present, there are 100 species of macrofungi belonged to 56 genera are used by the diverse tribes or people from various parts of India for the cure of various diseases (Table 1). Some of them are Pleurotus, Astraeus, Agaricus, Bovista, Coprinus, Pisolithus, Ganoderma, Lycoperdon, Trametes, Termitomyces, Phallus, Morchella, Lentinus, Lactarius, Hericium, Fomes, Flammulina and Auricularia which were highly used as a local medicine by the various tribes of India.

\section{TRADITIONAL USES OF WILD MUSHROOMS AS MEDICINE IN INDIA}

Wild mushrooms are used as herbal medicine, namely, wound healing, burning, itching, staunch bleeding eye disorders (pain, redness, conjunctivitis), fever and vomiting, typhoid, frost bites, ear puss, skin infections, curing stomach upset, brain tonic, against anger, constipation, weakness, eczema and mouth freshener (Devi, 2017; Dutta and Achariya, 2014; Kumar et al., 2014; 2017; Lahiri et al., 2010; Malik et al., 2017; Manna et al., 2014; Panda and Tayung, 2015; Rai et al., 1993; Thangaraj et al., 2017). Some mushrooms are used for a common illness, such as an antidepressant, treating lumbago, leg pains, numbness in limbs and tendon discomfort, nervous system colds, sore throats, sore eyes and stop bleeding (Malik et al., 2017; Pala et al., 2013; Panda and Tayung, 2015). Some major traditional uses of mushrooms against various scared diseases, viz, pneumonia, respiratory problems, asthma, jaundice, pox, goiters, diabetes, cancer, aphrodisiac, invigorative, revitaliser, anti-aging, kidney stones and partial paralysis (Kumar et al., 2014; 2017; Manna et al., 2014; Pala et al., 2013; Panda and Swain, 2011; Panda and Tayung, 2015; Rai et al., 1993). Wild mushrooms are also used by the tribal people for private diseases, such as aphrodisiac, menstrual cramps, to enhancing the lactation and prescribed to women after deliveries, treat male infertility and male visceral organ infections (Kumar et al., 2017; Thangaraj et al., 2017). Different ethnomedicinally important mushrooms with their traditional names of macrofungi, names of the tribes with their locality, purposes of use, various preparation techniques along with their references of publication are mentioned in Table 1.

\section{CONCLUSION}

Present finding concluded that wild mushrooms may contain various active compounds due to which they showed different medicinal behavior and used by the tribal peoples of the world. The traditional drug preparation knowledge of mushrooms could preferably attract scientist which will corporate to indulge their interest for the exploration of underlying scientific, economic and biological prospects. Accurate identification is also necessary because without any proper identification, we cannot explore traditional knowledge of wild mushrooms. Therefore, there is an urgent need to document indigenous knowledge about wild medicinal mushrooms which were used by the tribal peoples belonging to all the states of India as well as all over the world. This study will also be helpful for future researchers to identify the active compound of traditionally used wild macrofungi which were responsible for different medicinal activities. It is also needed to develop species specific cultivation of traditionally used mushrooms for commercialization and also for conservation. Many mushrooms species still remain unexplored and their medicinal, nutritional properties, as well as health benefits are unknown to us.

\section{ACKNOWLEDGMENTS}

The first author is thankful to the Department of Biotechnology (DBT), Government of India, for the financial assistance received through project (Sanctioned Order no. BT/463/ NE/TBP/2013).

\section{CONFLICT OF INTEREST}

The authors have declared that they have no conflict of interest.

\section{FUNDING}

Department of Biotechnology (DBT), Government of India (Sanctioned Order no. BT/463/NE/TBP/2013).

\section{ETHICS APPROVAL}

Not applicable.

\section{REFERENCES}

Adhikari MK, Devkota S, Tiwari RD. Ethnomycological knowledge on uses of wild mushrooms in Western and Central Nepal. Our Nature, 2005; 4:13-9.

Agrahar-Murugkar D, Subbulakshmi G. Nutritional value of edible wild mushrooms collected from the Khasi hills Meghalaya. Food Chem, 2005; 89:599-603.

Ayodele SM, Akpaja EO, Adamu Y. Some edible and medicinal mushrooms found in Igala land in Nigeria and their sociocultural and ethnomycological uses. In Proceeding of the 5th International Medicinal Mushroom Conference, Nantong, China, 2009, pp 526-31.

Baruah HK, Sing DK, Islam M. On the distribution of higher basidiomycetes in the Sibsagar district, India: Assam. Bull Bot Surv, 1971; 13(3\&4):285-9.

Basumatary M, Gogoi M. Uses of wild edible macro fungi by Bodo community of Kokrajhar district, Assam, India. Trop Plant Res, 2016; 3(1):176-81.

Boa E. Wild edible fungi, a global overview of their use and importance to people. Non-Wood Forest Products Series No. 17, FAO, Rome, Italy, 2004.

Chang ST, Buswell JA. Mushroom nutriceuticals. World J Microbiol Biotechnol 1996; 12(5):473-6.

Chang ST, Miles PG. Mushrooms: cultivation, nutritional value, medicinal effect, and environmental impact. 2nd edition, CRC Press, New York, NY, p 451, 2004.

Charaya MU, Mehrotra RS. From ethnomycology to fungal biotechnology: a historical perspective. In: Singh J, Aneja KR (eds.). From ethnomycology to fungal biotechnology: exploiting fungi from natural resources for novel products, Springer, New York, NY, pp 1-10, 1999.

Chauhan RS, Tiwari D, Bisht AS, Shukla A. Ex situ conservation of medicinal and aromatic plants in Bharsar, Uttarakhand, India. Med Plants, 2014; 6(4):282-92.

Devi K. Ethnomycological Studies of Mushrooms in Kamrup District of Assam. IJRCS, 2017; 1(7):50-6.

Devi K, Brahma J, Shrivastava K. Documentation of four hitherto unreported wild edible macro fungi from Chirang District of Assam, NorthEast India. Int J Conserv Sci, 2016; 7(3):709-18

Dutta A.K, Achariya K. Traditional and ethno-medicinal knowledge of mushrooms in West Bengal, India. Asian J Pharm Clin Res, 2014; 7(4):161-4. 
Hawksworth DL. The magnitude of fungal diversity: the 1.5 million species estimate revisited. Microbiol Res, 2001; 105:1422-32.

Hernández-Santiago F, Pérez Moreno J, Xoconostle Cázares B, Almaraz Suárez JJ, Ojeda Trejo E, Mata G, Díaz Aguilar I. Traditional knowledge and use of wild mushrooms by Mixtecs or Ñu savi, the people of the rain, from Southeastern Mexico. J Ethnobiol Ethnomed, 2016; $35(12): 1-22$.

Khaund P, Joshi SR. Wild edible macrofungal species consumed by Khasi tribe of Meghalaya, India. Ind J Nat Prod Resour, 2014; 4(2):197204.

Kimn H, Song MJ. Analysis of traditional knowledge for wild edible mushrooms consumed by residents living in Jirisan National Park (Korea). J Ethnopharmacol, 2014; 153:90-7.

Kumar M, Harsh NSK, Prasad R, Pandey VV. An ethnomycological survey of Jaunsar, Chakrata, Dehradun, India. JoTT, 2017; 9(9):10717-25.

Kumar R, Pandey S, Tapwal A, Rishi RR, Giri K, Mishra G. Ethnomycological Knowledge on Wild Mushrooms by tribes of Mokokchung, Nagaland, North East India. J Ethnobiol Tradit Med, 2014; 122:890-9.

Lahiri SS, Shukla MD, Shaha MB, Modic HA. Documentation and analysis of certain macrofungal traditional practices from WesternIndia (Gujarat). Ethnobot Leaflets, 2010; 14:626-41.

Lakhanpal TN, Shad O, Rana M. Biology of Indian morels. I K International Publishing, New Delhi, India, 2010.

Malik AR, Wani AH, Bhat MY, Parveen S. Ethnomycological knowledge of some wild mushrooms of northern districts of Jammu and Kashmir, India. Asian J Pharm Clin Res, 2017; 10(9):399-405.

Manna S, Ray D, Roy A. Tribal relation to spatio temporal variation of wild mushrooms in Eastern Lateritic Part of India. Ethnobot Res Appl, 2014; 12:015-24. $53: 916-7$

Myers N. Biodiversity hotspots revisited. Bioscience, 2003;

Okigbo RN, Nwatu CM. Ethnostudy and usage of edible and medicinal mushrooms in some parts of Anambra State, Nigeria. Nat Resour, 2015; 6:79-89.

Pala SA, Wani AH, Bhat MY. Ethnomycological studies of some wild medicinal and edible mushrooms in the Kashmir Himalayas (India). Int J Med Mushrooms, 2013; 15(2):211-20.

Panda AK, Swain KC. Traditional uses and medicinal potential of Cordyceps sinensis of Sikkim. J Ayurveda Integr Med, 2011; 2(1):9-13.

Panda MK, Tayung K. Documentation and ethnomedicinal knowledge on wild edible mushrooms among ethnic tribes of Northern Odisha, India. Asian J Pharm Clin Res, 2015; 8(4):139-43.

Paul M, Sarma TC, Sarma GC. Occurrence of some economically important macrofungi in Ultapani Reserve Forest under Manas Biosphere Reserve, Assam. Int J Adv Res, 2015; 3(9):319-25.
Purkayastha RP, Chandra A. Manual of Indian edible mushrooms. Today and Tomorrow's Printers and Publishers, New Delhi, India, 1985.

Rai BK, Ayachi SS, Rai A. A note on Ethno-myco-medicines from central India. Mycologist, 1993; 7:192-3.

Sachan SKS, Patra JK, Thatoi HN. Indigenous knowledge of ethnic tribes for utilization of wild mushrooms as food and medicine in similipal biosphere reserve, Odisha, India. Int J Agric Technol, 2013; 9(2):403-16.

Sarma TC, Sarma I, Patiri BN. Wild edible mushrooms used by some ethnic tribes of Western Assam. Bioscan, 2010; 10(3):613-25.

Sing NI, Sing SM, Th C. Fleshy fungi of Manipur. In: Vij SP, Kondo K, Sharma ML, Gupta A (eds.). Plant genetic diversity: exploaration, evaluation, conservation. Afficiated East West Press Pvt. Ltd., New Delhi, India, pp 9-13, 2002.

Sing NI, Sing SM. Wild Edible fleshy fungal flora of Manipur. Bioveel, 1993; 4(2):153-8.

Singh MN, Chhetry GKN. Biodiversity of macrofungi in Imphal, India-I. Indian Phytopath, 2010; 63(1):414-21.

Srivastava B, Dwivedi AK, Pandey VN. Ethnobotanical survey, distribution and utilization of Termitomyces species in Gorakhpur forest division. PSF, 2011; 1(3):28-33.

Tanti B, Lisha G, Sharma GC. Wild edible fungal resources used by ethnic tribes of Nagaland, India. Indian J Tradit Know, 2011; 10:512-5.

Thangaraj R, Raj S, Renganathan K. Wound healing effect of King Alfred's Mushroom (D.concentrica) used by Tribes of Sirumalai Hills, Tamilnadu, India. Int J Pharm Pharm Sci, 2017; 9(7);161-4.

Valverde ME, Hernández-Pérez T, Paredes-López O. Edible mushrooms: improving human health and promoting quality of life. Intl J Microbiol, 2015; 2015:1-14.

Wani BA, Bodha RH, Wani AH.. Nutrional and medicinal importance of mushrooms. J Med Plants Res, 2010; 4(24):2598-604.

Wasser SP. Medicinal mushroom science: History, current status, future trends, and unsolved problems. Int J Med Mushrooms, 2010; 12(1):1-16.

Wasson RG. Soma: divine mushroom of immortality, Ethnomycology Studies No.1, Harvest special, Harcourt Brace Jovanovich, New York, NY, 1971

WHO traditional medicine strategy 2002-2005. Document $\mathrm{WHO} / \mathrm{EDM} / \mathrm{TRM} / 2002$.

\section{How to cite this article:}

Debnath S, Debnath B, Das P, Saha AK. Review on an ethnomedicinal practices of wild mushrooms by the local tribes of India. J Appl Pharm Sci, 2019; 9(08):144-156. 